\title{
INrf2 (Keap1) targets Bcl-2 degradation and controls cellular apoptosis
}

\author{
SK Niture ${ }^{1}$ and AK Jaiswal ${ }^{*}, 1$
}

Cytosolic inhibitor of Nrf2 (INrf2) is an adaptor protein that mediates ubiquitination/degradation of NF-E2-related factor 2 (Nrf2), a master regulator of cytoprotective gene expression. In this paper, we demonstrate that INrf2 degrades endogenous antiapoptotic B-cell CLL/lymphoma 2 (Bcl-2) protein and controls cellular apoptosis. The DGR domain of INrf2 interacts with the $\mathrm{BH} 2$ domain of $\mathrm{Bcl}-2$ and facilitates INrf2:Cul3-Rbx1-mediated ubiquitination of $\mathrm{Bcl}-2$ by the conjugation of ubiquitin molecules to lysine17 of Bcl-2. Further studies showed that INrf2 enhanced etoposide-mediated accumulation of Bax, increased release of cytochrome $c$ from mitochondria, activated caspase-3/7, and enhanced DNA fragmentation and apoptosis. Antioxidants antagonized Bcl-2:INrf2 interaction, led to the release and stabilization of $\mathrm{Bcl}-2$, increased Bcl-2:Bax heterodimers and reduced apoptosis. Moreover, dysfunctional/mutant INrf2 in human lung cancer cells failed to degrade Bcl-2, resulting in decreased etoposide and UV/ $\gamma$ radiation-mediated DNA fragmentation. These data provide the first evidence of INrf2 control of Bcl-2 and apoptotic cell death, with implications in antioxidant protection, survival of cancer cells containing dysfunctional INrf2, and drug resistance.

Cell Death and Differentiation (2011) 18, 439-451; doi:10.1038/cdd.2010.114; published online 24 September 2010

The NF-E2-related factor 2 (Nrf2):cytosolic inhibitor of Nrf2 (INrf2) complex serves as a sensor of chemical/radiationinduced oxidative/electrophilic stress. ${ }^{1}$ Nrf2 resides predominantly in the cytoplasm, where it interacts with INrf2 (inhibitor of Nrf2) or Keap1 (Kelch-like ECH-associated protein). ${ }^{1}$ INrf2 functions as an adaptor protein for Cul3-Rbx1-dependent degradation of Nrf2. The N-terminal BTB domain of INrf2 binds to Cul3-Rbx1, whereas the C-terminal Kelch (DGR) domain interacts with Nrf2 and facilitates Nrf2 ubiquitination and degradation, resulting in the repression of Nrf2-dependent gene expression. ${ }^{2-4}$ Oxidative or electrophilic stress generated from chemicals or radiation modifies reactive cysteines of INrf2 (C151, C272, and C288), followed by PKC $\delta$-mediated phosphorylation of Nrf2S40, which leads to dissociation of Nrf2 from INrf2 and increased transcription of Nrf2-dependent genes. ${ }^{5-7}$

The induction of Nrf2 downstream genes encodes cytoprotective proteins that neutralize reactive molecules, eliminate damaged macromolecules, reduce inflammation, and restore cellular redox homeostasis. ${ }^{8,9} \mathrm{Nrf2}$ is switched 'ON' in response to stress by mechanisms as described above. Nrf2 is switched 'OFF' once the stress subsidizes by a distinct mechanism. Tyrosine kinase Fyn phosphorylates Nrf2Y568, leading to nuclear export and degradation of Nrf2. ${ }^{10,9}$ The switching 'ON' and 'OFF' of Nrf2 protects cells and promotes cell survival.

INrf2-null mice demonstrated persistent accumulation of Nrf2 in the nucleus that led to postnatal death from malnutrition resulting from hyperkeratosis in the esophagus and forestomach. ${ }^{11}$ Reversed phenotype of INrf2 deficiency by breeding with Nrf2-null mice suggested that tightly regulated negative feedback might be essential for cell survival. ${ }^{8}$ The systemic analysis of INrf2 genomic locus in human lung-cancer patients and cell lines showed that deletion, insertion, and missense mutations in functionally important domains of INrf2 result in reduction of INrf2 affinity for Nrf2 and elevated expression of cytoprotective genes. ${ }^{12,13}$ Unrestrained activation of Nrf2 in cells increases the risk of adverse effects, including survival of damaged cells, tumorigenesis, and drug resistance. ${ }^{9}$ Therefore, it appears that cells contain mechanisms that autoregulate cellular abundance of Nrf2. ${ }^{14}$ In other words, Nrf2 induces transcription of INrf2 for self-destruction.

Structural and functional analyses of INrf2 have identified a Kelch (DGR) domain, which interacts with several proteins. Although Nrf2 is a well-known substrate for INrf2, the DGR domain of INrf2 has been reported to bind other proteins including Nrf1, prothymosin- $\alpha$, fetal Alz clone 1, and IKK $\beta .^{15-18}$ It is noteworthy that binding of a protein with the INrf2 DGR region does not always lead to degradation of the protein. Recently, we have shown that prothymosin- $\alpha$ interacts with the DGR domain of INrf2 and this interaction is required for nuclear localization of INrf2. ${ }^{1}$ Therefore, INrf2 and its interacting partners have several different roles in cell signaling and survival.

The B-cell CLL/lymphoma 2 (Bcl-2) family of proteins regulates cell death and survival. ${ }^{19,20} \mathrm{Bcl}-2$ proteins are

\footnotetext{
${ }^{1}$ Department of Pharmacology and Experimental Therapeutics, University of Maryland School of Medicine, Baltimore, MD, USA

*Corresponding author: A Jaiswal, Department of Pharmacology and Experimental Therapeutics, University of Maryland School of Medicine, 655 West Baltimore Street, Baltimore, MD 21201, USA. Tel: + 410706 2285; Fax: + 410706 5692; E-mail: ajaiswal@som.umaryland.edu

Keywords: Nrf2; INrf2 (Keap1); Bcl-2; Anti-apoptotic; Apoptosis

Abbreviations: Bcl-2, B-cell CLL/lymphoma 2; Nrf2, NF-E2-related factor 2; INrf2, cytosolic inhibitor of Nrf2, also known as Keap1; ARE, antioxidant response element; NQ01, NAD(P)H:quinine oxidoreductase 1; t-BHQ, tert-butylhydroquinone; IP, immunoprecipitation; GFP, green fluorescent protein; HA, hemagglutinin; HRP, horseradish peroxidase; siRNA, small interfering RNA; DAPI, 4',6-diamidino-2-phenylindole; PBS, phosphate-buffered saline; FITC, fluorescein isothiocyanate; WB, western blotting or immunoblotting

Received 29.1.10; revised 16.7.10; accepted 02.8.10; Edited by J Silke; published online 24.9.10
} 
central regulators of caspase activation, and have a key role in cell death by regulating the integrity of the mitochondrial and endoplasmic reticulum membranes. ${ }^{21,22}$ The $\mathrm{Bcl}-2$ family of proteins is classified into three subfamilies. The $\mathrm{Bcl}-2$ subfamily includes Bcl-2, Bcl-xL, and Bcl-w, all of which exert anticell death activity and share sequence homology, particularly within four regions, $\mathrm{BH}$ (Bcl-2 homology) 1-4 domains. The Bax subfamily consists of Bax and Bak, which contain $\mathrm{BH} 1, \mathrm{BH} 2$, and $\mathrm{BH} 3$ homology domains but lack the $\mathrm{BH} 4$ domain and are proapoptotic. The Bik subfamily that includes Bik and Bid contains only the $\mathrm{BH} 3$ domain and lack $\mathrm{BH} 1, \mathrm{BH} 2$, and $\mathrm{BH} 3$ domains. Bik members are proapoptotic. One of the important features of $\mathrm{Bcl}-2$ proteins is that they have the ability to form homodimers and heterodimers. The life or death of a cell may be determined by the Bcl-2 family of proteins in two ways, either through heterodimerization between antiapoptotic and proapoptotic members, or through the independent functions of these proteins. In either case, the ratio between antiapoptotic and proapoptotic members of the Bcl-2 family may determine the susceptibility of a cell to apoptosis.

In this report we demonstrate a novel mechanism of the control of Bcl-2 and apoptotic cell death. We show that the INrf2:Cul3-Rbx1 complex ubiquitinates Bcl-2 lysine17 residue and degrades Bcl-2. Specifically INrf2, through its DGR region, interacts with the $\mathrm{BH} 2$ domain of $\mathrm{Bcl}-2$ and facilitates $\mathrm{Bcl}-2$ ubiquitination and degradation. We also show that INrf2mediated degradation of $\mathrm{Bcl}-2$ leads to decreased $\mathrm{Bcl}-2$ :Bax heterodimers, resulting in an increased level of Bax, and subsequently enhanced etoposide and radiation $(\mathrm{UV} / \gamma)$ mediated apoptosis in cancer cells. We further show that antioxidants antagonize INrf2:Bcl-2 interaction, leading to the stabilization of $\mathrm{Bcl}-2$ and cell survival.

\section{Results}

INrf2 mediates ubiquitination and degradation of antiapoptotic factor Bcl-2. Flag-INrf2-HEK293 cells expressing tetracycline-inducible Flag-INrf2 were developed. Immunoprecipitation of INrf2 with Flag antibody, followed by mass spectrometry analysis, revealed that Flag-INrf2 interacted with antiapoptotic protein Bcl-2 (Supplementary Figure S1). Therefore, we investigated the role of INrf2 in control of Bcl-2 and apoptotic cell death. The transfection of mouse hepatoma (Hepa-1) cells with small interfering (si)RNA showed dose-dependent silencing of INrf2 expression (Figure 1a). The silencing of INrf2 led to a dose-dependent increase in $\mathrm{Bcl}-2$ and decrease in proapoptotic protein Bax (Figure 1a). In the same experiment, the level of Nrf2 also increased as expected (Figure 1a). In a related experiment, overexpression of INrf2-V5 protein in Hepa-1 cells showed INrf2-V5-dependent decrease in Bcl-2 and Nrf2 protein levels, and increase in Bax (Figure 1b). Next, we determined the effect of siRNA-mediated inhibition and cDNA-derived overexpression of INrf2 on Bcl-2 transcription (Figure 1c). Interestingly, silencing of endogenous INrf2 or overexpression of INrf2-V5 protein by transfection in Hepa- 1 cells led to an $\sim 10 \%$ increase or decrease of $\mathrm{Bcl}-2$ mRNA levels, respectively (Figure 1c). These results suggested that INrf2 regulates $\mathrm{Bcl}-2$ protein mostly by degradation and partly through Nrf2-regulated transcription of $\mathrm{Bcl}-2$. Further support for this conclusion was obtained by analyzing Bcl-2 and Bax content in HEK293 (control) and INrf2-293 cells expressing tetracycline-inducible Flag-INrf2 (Figure 1d). On treatment with tetracycline, INrf2-293 cells but not control 293 cells showed time-dependent increase in Flag-INrf2. This led to a decrease in endogenous Bcl-2 and an increase in Bax (Figure 1d, left two panels). Similar results were also observed for Bcl2-V5 in transfected INrf2-293 cells (Figure 1d, right panel). The treatment with MG132 inhibited INrf2-mediated degradation of endogenous and transfected $\mathrm{Bcl}-2$, leading to $\mathrm{Bcl}-2$ stabilization (Figure 1d). The decrease in $\mathrm{Bcl}-2$ was due to increased ubiquitination of endogenous and transfected $\mathrm{Bcl}-2$ in Flag-INrf2-overexpressing cells (Figure 1e-endogenous/1f-transfected). The treatment with proteasome inhibitor MG132 further increased the ubiquitination of Bcl-2 and stabilized Bcl-2 in INrf2-293 cells (Figure 1g). These results together suggested that INrf2 controls $\mathrm{Bcl}-2$ by regulating ubiquitination and degradation.

The INrf2:Cul3-RBX1 complex ubiquitinates Bcl-2 at the $\mathrm{N}$-terminal lysine17 in vivo and in vitro. To examine the involvement of the INrf2-Cul3-RBX1 complex in the ubiquitination and degradation of $\mathrm{Bcl}-2$, Hepa-1 cells were transfected with INrf2 or Cul3 siRNA and Bcl2 ubiquitination was analyzed. The results demonstrated that siRNA inhibition of INrf2 or Cul3 led to a significant decrease in ubiquitination and an increase in the stabilization of Bcl-2 (Figure $2 \mathrm{a}$ and $\mathrm{b}$ ). In addition, overexpression of Myc-Rbx1, hemagglutinin (HA)-Cul3, or Flag-INrf2, individually or combined, degraded cellular Bcl-2 (Figure 2c, left panel) because of increase in ubiquitination of $\mathrm{Bcl}-2$ (Figure 2c, right panel). Interestingly, overexpression of INrf2 had a higher magnitude of effect, presumably because of its function as an adaptor between $\mathrm{Bcl}-2$ and Cul3-Rbx1 (Figure 2c). The analysis of mouse $\mathrm{Bcl}-2$ amino acid sequence showed the presence of only four lysine residues at amino acid positions $17,22,214$, and 236 that were found to be highly conserved in humans and rats (Figure 2d). Mouse Bcl-2K17, K22, K214, and K236 were individually mutated to alanine to identify the target lysine(s) of INrf2-mediated ubiquitination. Wild-type and mutant $\mathrm{Bcl}-2$ were analyzed for endogenous INrf2mediated degradation and ubiquitination in Hepa-1 cells (Figure 2e, upper and lower panels). Only the Bcl2K17A mutant, and not other mutants, showed stabilization (Figure $2 \mathrm{e}$, upper panel) and significantly reduced ubiquitination (Figure 2e, lower panel). To examine the INrf2mediated specific ubiquitination of wild-type Bcl-2 and $\mathrm{Bcl}-2$ lysine mutants, we used 293 cells and INrf2-293 cells. Cells were co-transfected with Bcl-2-V5 or lysine mutants and $\mathrm{HA}$ ubiquitin for $24 \mathrm{~h}$. Thereafter, one set of cells treated with tetracycline for $12 \mathrm{~h}$ and $\mathrm{Bcl}-2$ ubiquitination was analyzed. The results demonstrate that the overexpression of FlagINrf2 significantly increased Bcl-2 ubiquitination (2.5-fold) (Figure 2f). Further, the Bcl-2K17A mutant showed significantly (greater than $90 \%$ ) decreased ubiquitination in INrf2-293 cells (Figure 2f, right panel). These data also suggested that $\mathrm{K} 17$ of $\mathrm{Bcl}-2$ is the major site of INrf2: Cul3-Rbx1-mediated ubiquitination of $\mathrm{Bcl}-2$. This conclusion was further supported by in vitro studies (Supplementary 
a

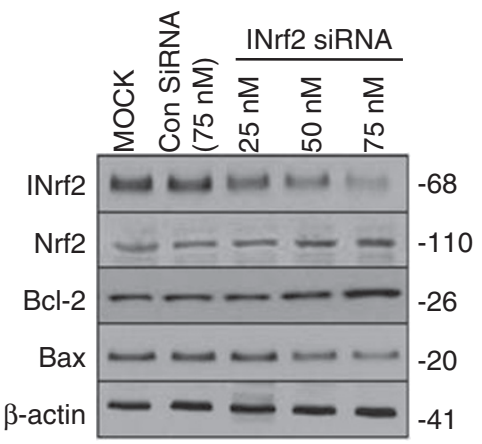

b

$\begin{array}{lllllll}\text { pcDNA } & 0.8 & 0.6 & 0.4 & 0.2 & 0 & \mu \mathrm{g}\end{array}$ $\begin{array}{llllllll}\text { INrf2-V5 } & 0.0 & 0.2 & 0.4 & 0.6 & 0.8 & \mu \mathrm{g}\end{array}$

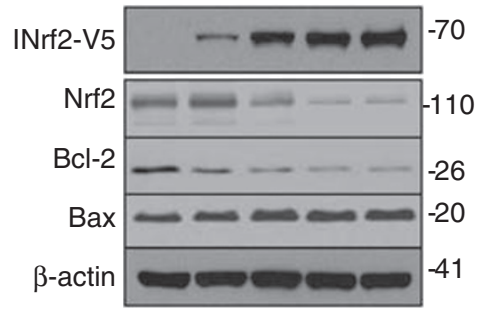

c

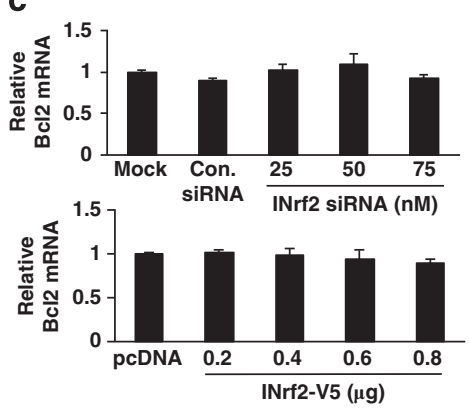

d

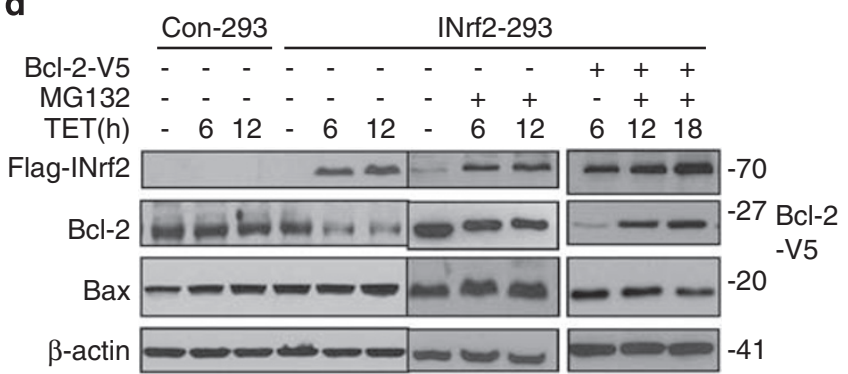

f

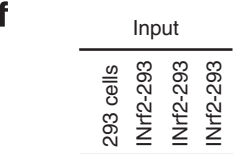

TET h $\begin{array}{llll}18 & 6 & 12 & 18\end{array}$

$\mathrm{Bcl} 2-\mathrm{His}++++$

$\mathrm{HA}-\mathrm{UB}++++$
$\mathrm{Bcl} 2-\mathrm{His} \sim \ldots-27$

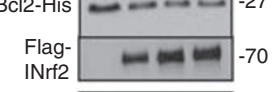

$\beta$-actin -41
PAG NiNTABDS

P-AG Ni-NTA Beads P-AG Ni-NTA Beads

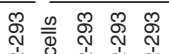

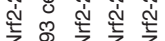

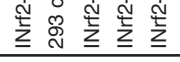

TET $\begin{array}{lllll}18 & 18 & 6 & 12 & 18\end{array}$

$\mathrm{Bcl} 2-\mathrm{His}+++++$
$\mathrm{HA}-\mathrm{UB}+++++$

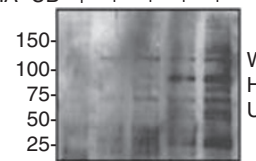

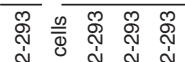

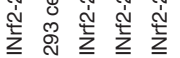

$181861218 \mathrm{~h}$

$++++$

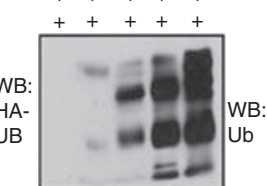

e

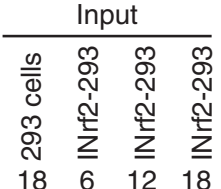

$\begin{array}{lllll}\text { Tet (h) } & 18 & 6 & 12 & 18\end{array}$

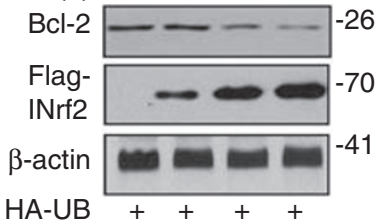

HA-UB

\section{g}

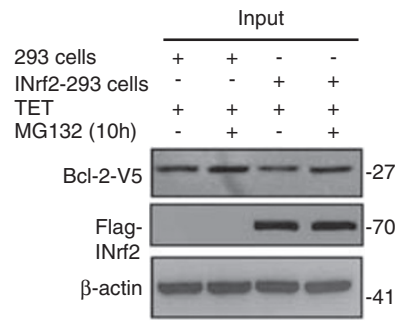

Figure 1 INrf2 regulates ubiquitination and degradation of Bcl-2. (a) Immunoblot analysis. Hepa-1 cells transfected with increasing concentrations of INrf2 siRNA were lysed and immunoblotted with indicated antibodies. (b) Immunoblot analysis. Hepa-1 cells transfected with increasing concentrations of pcDNA-INrf2-V5 plasmid were lysed and immunoblotted with indicated antibodies. (c) RT-PCR analysis. Hepa-1 cells were transfected with either increasing concentrations of INrf2siRNA (upper panel) or increasing amounts of INrf2-V5 plasmid (lower panel) for $24 \mathrm{~h}$. Cells were harvested and Bcl-2 mRNA was quantified by real-time PCR. (d) Immunoblot analysis of INrf2mediated degradation of endogenous and transfected Bcl-2. Control 293 or INrf2-293 cells were treated with tetracycline $(0.5 \mu \mathrm{g} / \mathrm{ml})$ or pretreated with MG132 $(5 \mu \mathrm{M})$ as shown in figures and $60 \mu \mathrm{g}$ of cell lysates was immunoblotted with indicated antibodies (left two panels). In a related experiment, INrf2-293 cells were transfected to overexpress Bcl2-V5, treated with tetracycline and immunoblotted with indicated antibodies. (e) Immunoblot analysis of INrf2-mediated Bcl-2 ubiquitination. Control 293 and INrf2-293 cells were transfected with HA-ubiquitin and treated with tetracycline $(0.5 \mu \mathrm{g} / \mathrm{ml})$ for $6-18 \mathrm{~h}$. Cell lysates $(60 \mu \mathrm{g})$ were immunoblotted with indicated antibodies (left input panel). A volume of $1 \mathrm{mg}$ of the same cell lysates was immunoprecipitated with anti-Bcl-2 antibody and immunoblotted with anti-HA antibody (right panel). (f) Immunoblot analysis of INrf2-mediated ubiquitination of Bcl-2. Control 293 or INrf2-293 cells were co-transfected with HA-ubiquitin and pcDNA3.1-Bcl2-V5-His-tagged plasmid construct for $24 \mathrm{~h}$ and cells were treated with tetracycline as indicated. Cell lysates $(60 \mu \mathrm{g})$ were immunoblotted with indicated antibodies (left panels) as input. A volume of $1 \mathrm{mg}$ of the same cell lysates was mixed with 50 ul of protein A/G beads (P-AG) or Ni-NTA resin. His-tagged Bcl-2 was pooled down and immunoblotted with anti-HA antibody (middle panel) or antiubiquitin antibody (right panel). (g) The effect of proteasome inhibitor MG132 on transfected Bcl-2-V5 ubiquitination in control 293 and INrf2-293 cells. Control 293 or INrf2-293 cells were co-transfected with HA-ubiquitin and Bcl2-V5 construct for $24 \mathrm{~h}$ and cells were treated with MG132 $(5 \mu \mathrm{M})$ for $10 \mathrm{~h}$ and later treated with tetracycline for $12 \mathrm{~h}$. Cell lysates $(60 \mu \mathrm{g})$ were immunoblotted with indicated antibodies (left panels) as input. A volume of $1 \mathrm{mg}$ of the same cell lysates was immunoprecipitated with anti-V5 antibody and western blotted with anti-HA antibody (right panel). All experiments were repeated three times and one representative set of data is shown

Figure S2). Hepa-1 cell extracts overexpressing INrf2: Cul3-Rbx1 proteins ubiquitinated and degraded in vitro transcribed/translated wild-type Bcl2-V5 and lysine mutants K22A-V5, K214A-V5, and K236A-V5, but not mutant Bcl-2K17A-V5 (Supplementary Figure S2a-c). Hepa-1 extract overexpressing INrf2:Cul3-Rbx1 also showed ubiquitination and degradation of bacterially purified $\mathrm{Bcl}-2$ (Supplementary Figure S2d).
INrf2 through its DGR domain interacts with the $\mathrm{BH} 2$ domain of Bcl-2. INrf2-mediated ubiquitination/degradation of $\mathrm{Bcl}-2$ and the regulation of $\mathrm{Bcl}-2$ stability suggested that INrf2 and $\mathrm{Bcl}-2$ interact with each other. The experiments investigated interaction of endogenous INrf2 and Bcl-2 in Hepa-1 cells (Figure 3a), interaction of tetracycline-induced Flag-INrf2 with endogenous Bcl-2 in INrf2-293 cells (Figure $3 b$ ), and interaction of Flag-INrf2 with Bcl-2-V5 in 
transfected INrf2-293 cells (Figure 3c). Anti-INrf2 antibodies immunoprecipitated INrf2, and Bcl-2 was pulled down with it (Figure 3a, upper two panels). Similarly, in reverse immunoprecipitation (IP), anti-Bcl-2 antibodies immunoprecipitated $\mathrm{Bcl}-2$ and INrf2 (Figure 3a, lower two panels). These results demonstrated interaction of endogenous INrf2 with Bcl-2.
In addition, immunoprecipitation of Flag-INrf2 pulled down endogenous $\mathrm{Bcl}-2$ and vice versa in INrf2-293 cells, but not in control 293 cells (Figure 3b). Furthermore, immunoprecipitation of Flag-INrf2 pulled down Bcl-2-V5 from HEK INrf2-293 cells transfected with Bcl-2-V5 cells (Figure 3c, top two panels) and vice versa (Figure $3 c$, lower two panels).

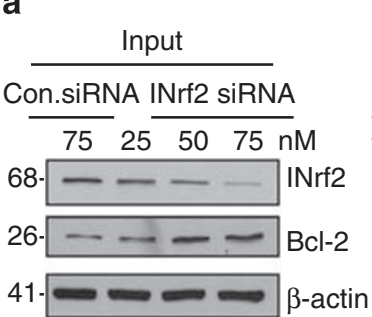

C

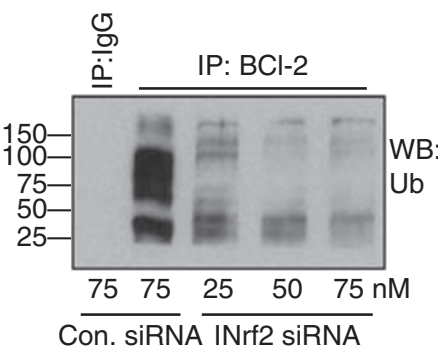

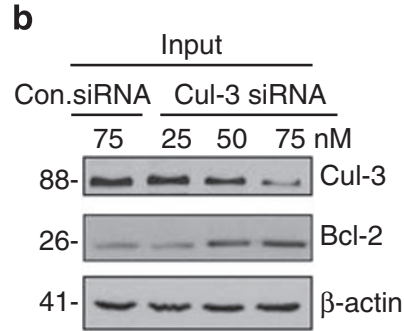

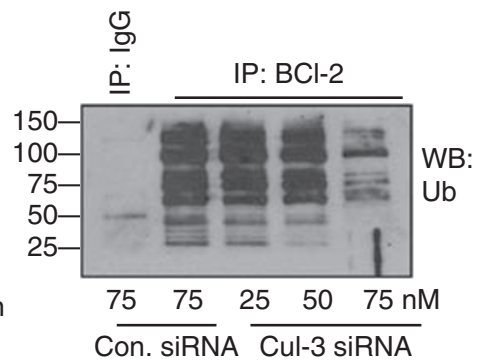

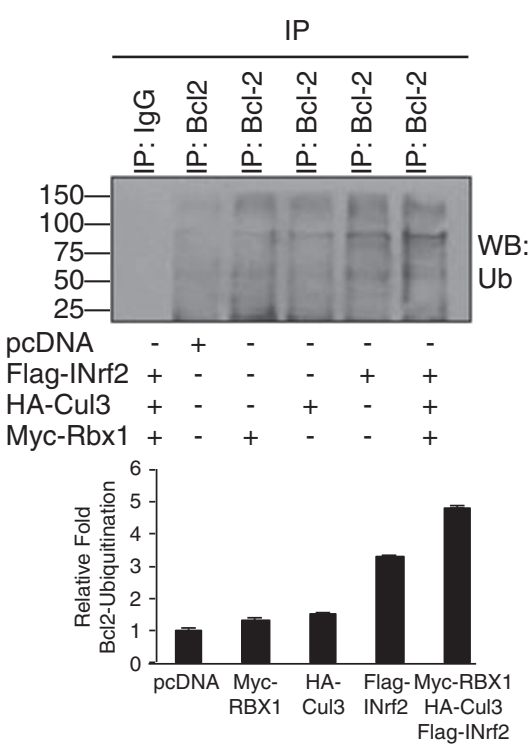

d

hBcl-2-nreivmkyihyklsqr...wlslktl ....Ighk mBcl-2-nreivmkyihyklsqr...wlslktl....Ighk rBcl-2-nreivmkyihyklsqr....wlslktll...lghk

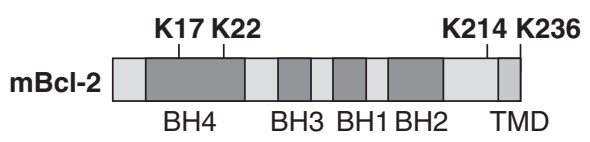

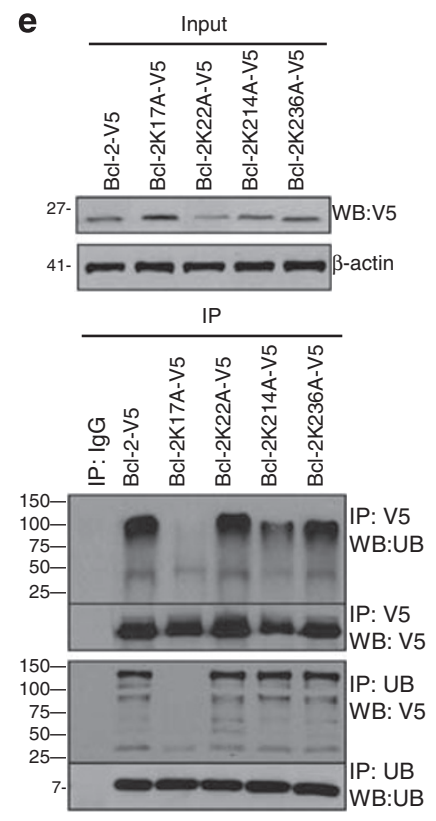

f

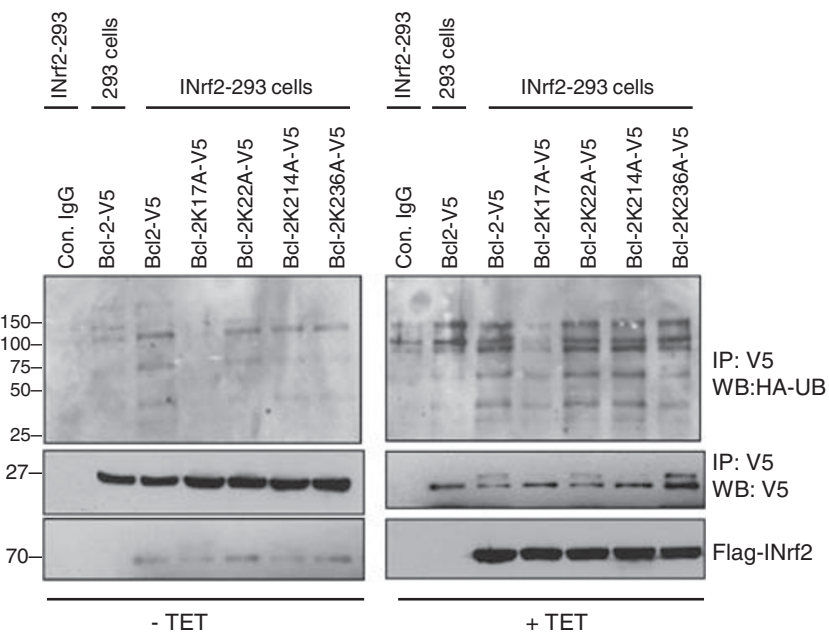


a

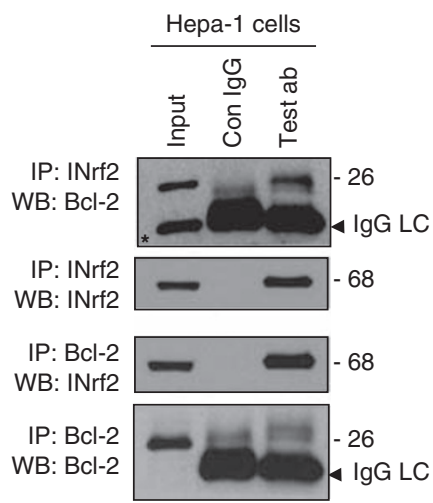

b

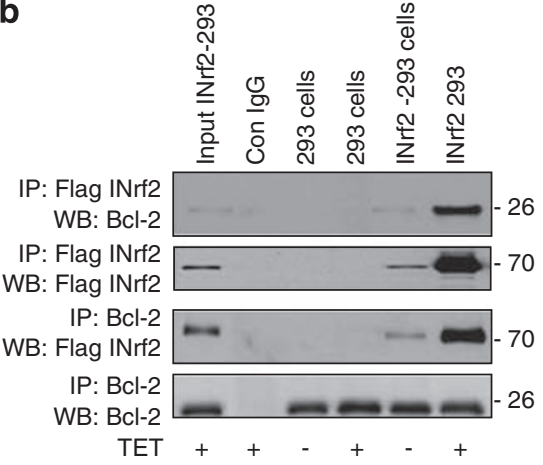

C

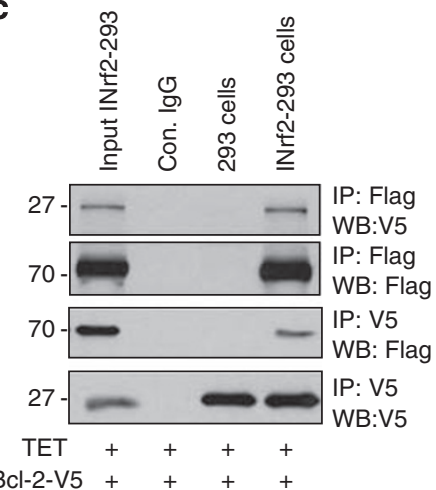

d

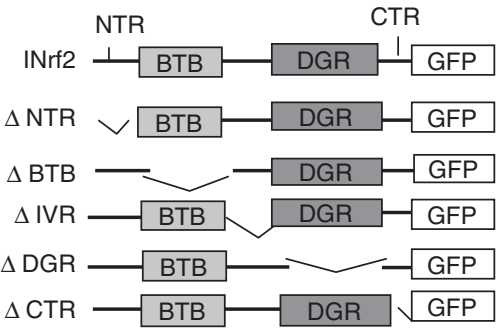

e

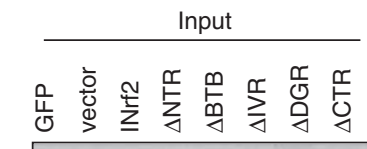

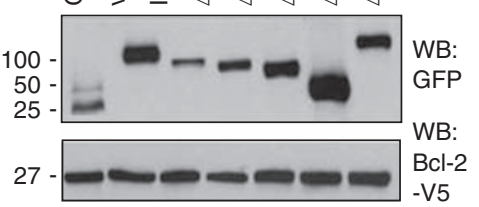

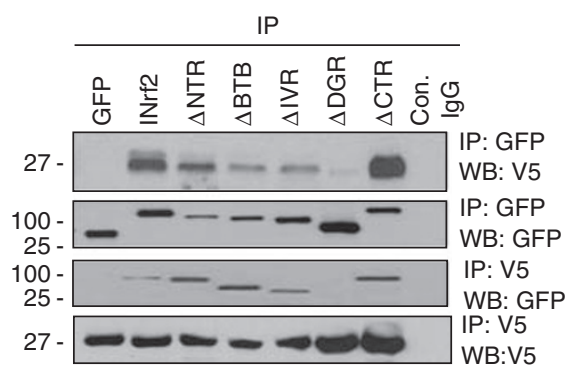

f

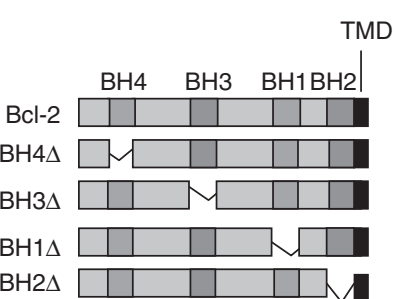

g

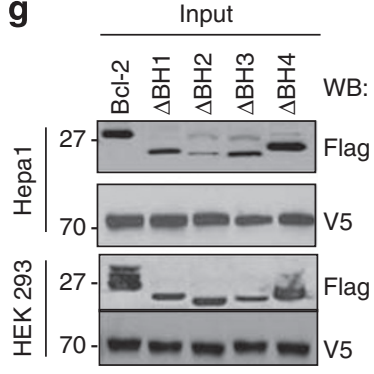

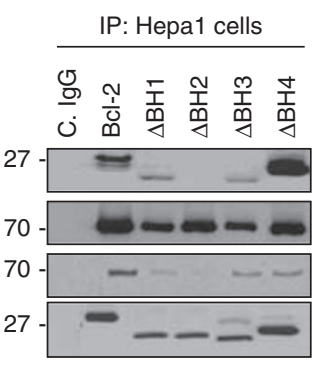

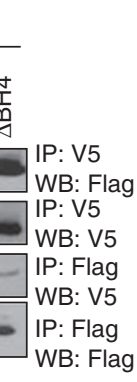

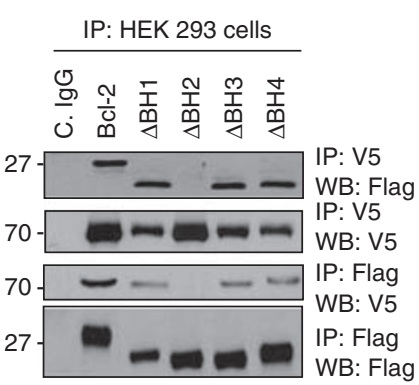

Figure 3 The INrf2 DGR domain interacts with the BH2 domain of Bcl-2. (a) IP/immunoblot analysis of INrf2:Bcl-2 interaction. Forward and reverse IP, followed by immunoblotting, analyzed endogenous INrf2:Bcl-2 interaction in Hepa1 cells. *Nonspecific band detected by anti-Bcl-2 antibody in left input lane. (b) Flag-INrf2 interaction with endogenous Bcl-2. Forward/reverse IP and immunoblot assays analyzed control 293 and INrf2-293 cells after treatment of cells with tetracycline $(0.5 \mu \mathrm{g} / \mathrm{ml})$ for $12 \mathrm{~h}$. (c) Flag-INrf2 interaction with Bcl-2-V5. IP/immunoblot analysis of control 293 or INrf2-293 cells after transfection with Bcl-2-V5 plasmid was performed. (d) Schematic presentation of INrf2 domain deletion mutants. (e) The INrf2-DGR domain required for interaction with Bcl-2. Hepa-1 cells were co-transfected with Bcl-2-V5 and indicated GFP-INrf2 domain deletions and the interaction of Bcl-2-V5 and GFP-INrf2 deletion domains were analyzed by IP/immunoblotting. (f) Schematic presentation of Bcl-2 deletion domains. (g) The Bcl-2 BH2 domain is essential for interaction with INrf2. Hepa-1 or HEK-293 cells were co-transfected with INrf2-V5 and Flag-Bcl-2 domain deletions, and the interaction of INrf2-V5 and Flag-Bcl-2 domain deletions was analyzed by IP/immunoblotting. (h) Amino acid sequence alignment of the BH2 domain from human, mouse, rat, and chicken Bcl-2. The amino acid residues tryptophan185, glycine190, and glycine191 are highly conserved in the Bcl-2 family of proteins. (i) Mutation of Bcl-2glycine190/191 leads to significantly decreased interaction of Bcl-2 with INrf2. Hepa-1 and HEK-293 cells were co-transfected with wild-type Bcl-2-V5 or mutant Bcl-2W185AV5 or Bcl-2G190AG191A-V5 plasmid, along with Flag-INrf2, for $24 \mathrm{~h}$ and lysates were immunoblotted with indicated antibodies as input (left panel). A volume of $1 \mathrm{mg}$ of same cell lysates from transfected Hepa-1 cells or HEK293 cells was immunoprecipitated with anti-V5 or anti-Flag antibody and immunoblotted with indicated antibodies. All experiments were repeated three times and one representative set of data is shown

Figure 2 The INrf2:Cul3-RBX1 complex is required for Bcl-2K17 ubiquitination. (a, b) siRNA inhibition of INrf2 or Cul3 reduces Bcl-2 ubiquitination. Hepa-1 cells were transfected with siRNA against INrf2 (a) or Cul3 (b) and lysates were immunoblotted with antibodies as indicated in input panels ( $\mathbf{a}$ and $\mathbf{b}$, left panels). For Bcl-2 ubiquitination, $1 \mathrm{mg}$ of the same cell lysates was immunoprecipitated with anti-Bcl-2 antibody and immunoblotted with antiubiquitin antibody (a and $\mathbf{b}$ right panels). (c) The INrf2:Cul3-Rbx1 complex ubiquitinates and degrades Bcl-2. Hepa-1 cells were transfected with Flag-INrf2 or HA-Cul3 or Myc-Rbx1, separately or in combination, and lysates were immunoblotted with antibodies as shown in input left panel. Same lysates $(1 \mathrm{mg})$ were immunoprecipitated with anti-Bcl-2 antibody and immunoblotted with antiubiquitin antibodies (right panel). (d) Amino acid sequence alignment of human, mouse and rat Bcl-2 protein. The four lysine residues are shown. (e) Bcl2K17 is required for Bcl-2 ubiquitination. Immunoblot analysis of Hepa-1 cells transfected with V5-tagged wild-type Bcl-2 and lysine mutants of Bcl-2 proteins for $24 \mathrm{~h}$. A volume of $60 \mu \mathrm{g}$ of cell lysates was immunoblotted with indicated antibodies (upper panels) as input. A volume of $1 \mathrm{mg}$ of the same cell lysates was immunoprecipitated with anti-V5 or antiubiquitin antibody and immunoblotted with indicated antibodies (lower panel). (f) INrf2-mediated ubiquitination of Bcl-2 and mutant proteins. Two sets of control 293 cells and INrf2-293 cells were co-transfected with Bcl-2-V5 or lysine mutants of Bcl-2, as indicated in figures, with $\mathrm{HA}$-ubiquitin for $24 \mathrm{~h}$ and then one set of cells was treated with tetracycline for $12 \mathrm{~h}$ and another without tetracycline. A volume of $1 \mathrm{mg}$ of cell lysates was immunoprecipitated with anti-V5 antibody and immunoblotted with anti-HA and anti-V5 antibody. The expression of Flag-INrf2 is presented in the lower immunoblot panel. All experiments were repeated three times and one representative set of data is shown 

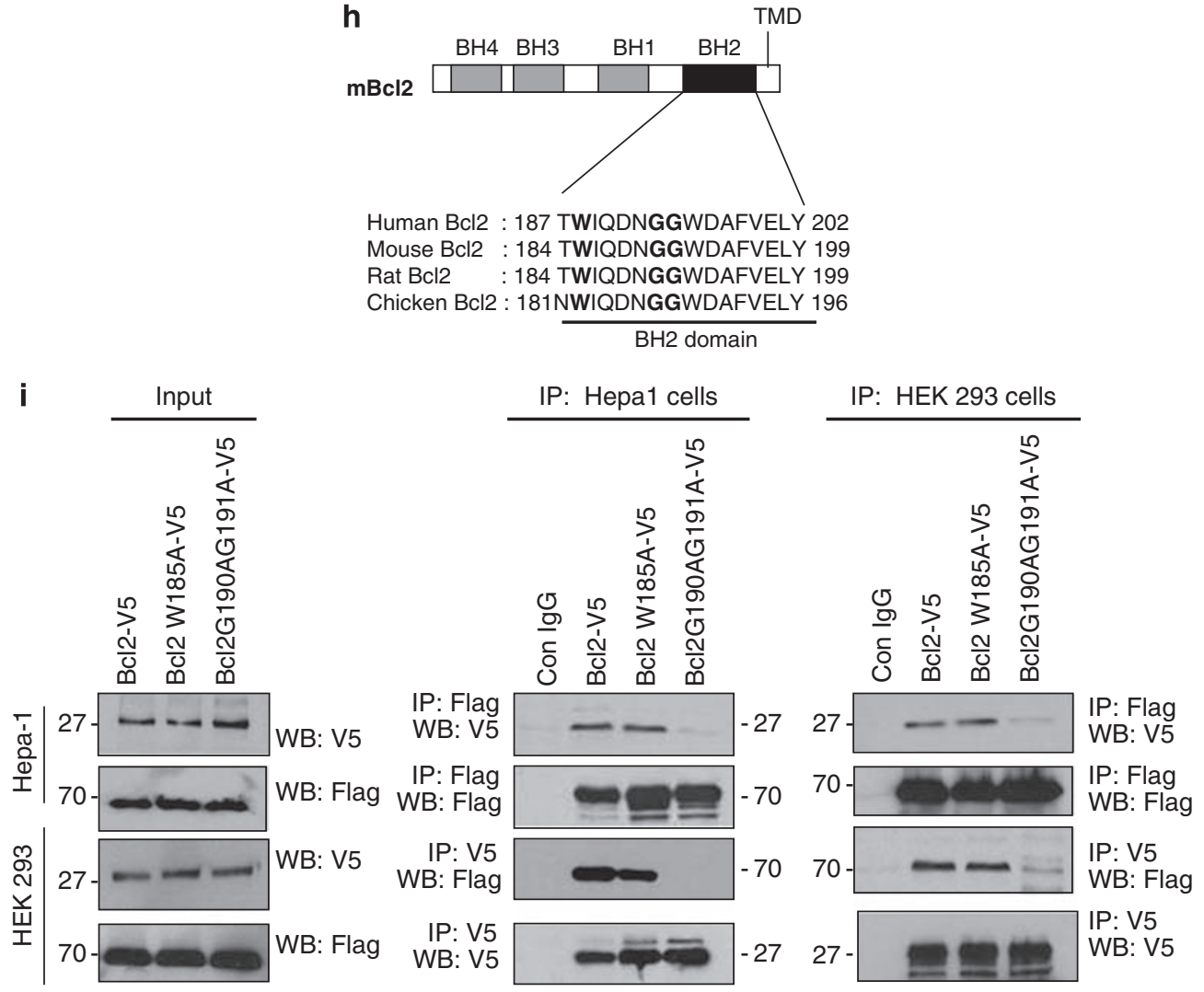

Figure 3 Continued

Next, we mapped the interacting domains of INrf2 and Bcl-2 proteins. INrf2 possesses five discrete domains designated as NTR (N-terminal region; 1-60 amino acids), BTB (broad complex, tamtrac, and bric-a-bric; 61-178 amino acids), IVR (linker region; 179-314 amino acids), DGR/Kelch (di-glycine repeats; 315-598 amino acids), and CTR (C-terminal region; 599-624 amino acids) (Figure 3d). We deleted the individual domains of INrf2 as described previously to generate INrf2 deletion mutants (Figure $3 \mathrm{~d}$ ). ${ }^{1}$ Hepa- 1 cells were co-transfected with INrf2 deletion mutants in separate experiments with Bcl-2-V5 and cell lysates were immunoblotted with anti-green fluorescent protein (GFP) and anti-V5 antibodies (Figure 3 e, left panel). The various INrf2 deletions and Bcl-2V5 proteins showed expression in Hepa-1 cells (Figure 3e, left panel). Same cell lysates were immunoprecipitated and immunoblotted with anti-GFP and anti-Flag antibodies (Figure $3 e$, right panel). The results from forward and reverse immunoprecipitation demonstrated that all deletion mutants except $\triangle$ DGRINrf2-GFP interacted with Bcl-2 (Figure 3e, right panels). This suggested that the DGR region of INrf2 is required for $\mathrm{Bcl}-2$ interaction. Further deletions in the DGR region showed that the entire DGR region of INrf2 is required for interaction with $\mathrm{Bcl}-2$ (Supplementary Figure S3a and b). Further, the $\mathrm{Bcl}-2$ protein consists of four unique $\mathrm{BH}$ domains that include $\mathrm{BH} 4, \mathrm{BH} 3, \mathrm{BH} 1$, and $\mathrm{BH} 2$, and a transmembrane domain (TMD) (Figure 3f). We generated $\mathrm{Bcl}-2$ deletion mutants deficient in the various $\mathrm{BH}$ domains to investigate which $\mathrm{BH}$ domain of $\mathrm{Bcl}-2$ is required for interaction with INrf2 (Figure 3f). Hepa-1 and HEK-293 cells were separately co-transfected with domain deletion mutants of Bcl-2 and INrf2-V5 and immunoblotted (Figure 3g, left panel). The various Flag-tagged domain deletions of $\mathrm{Bcl}-2$ and INrf2-V5 demonstrated expression in Hepa-1 and HEK293 cells (Figure 3g, left panel). Forward and reverse immunoprecipitation with anti-Flag and anti-V5 antibodies demonstrated that deletion mutant $\mathrm{Bcl}-2 \Delta \mathrm{BH} 2$ failed to interact with INrf2-V5 in both Hepa-1 and HEK293 cells (Figure $3 \mathrm{~g}$, middle and right panels). All other $\mathrm{Bcl}-2$ deletion mutants showed interaction with INrf2-V5. These results suggested that the $\mathrm{BH} 2$ domain of $\mathrm{Bcl}-2$ is required for $\mathrm{Bcl}-2$ interaction with INrf2. This conclusion was further supported with point mutations of the $\mathrm{BH} 2$ domain of $\mathrm{Bcl}-2$. The $\mathrm{BH} 2$ domain is highly conserved among human, mouse, rat, and chicken $\mathrm{Bcl}-2$ proteins (Figure $3 \mathrm{~h}$ ). The alignment of the Bcl-2 BH2 domain from different species also showed that tryptophane185 and double glycine190/191 are conserved across species (Figure $3 \mathrm{~h}$ ). We generated two mutations, W185A and double glycine mutant G190AG191A of Bcl-2, and their interaction with Flag-INrf2 was examined. The results demonstrate that wild-type $\mathrm{Bcl}-2$ and $\mathrm{Bcl}-2 \mathrm{~W} 185 \mathrm{~A}$ mutant interacted with Flag-INrf2 (Figure 3i); however, double glycine mutant Bcl-2G190AG191A demonstrated significantly reduced interaction with Flag-INrf2 in both Hepa-1 and HEK293 cells (Figure 3i, right panels). These observations supported our results from the deletion mutants that the $\mathrm{BH} 2$ domain of $\mathrm{Bcl} 2$ is required for INrf2 interaction (Figure 3i). The requirement of INrf2 DGR domain and $\mathrm{Bcl}-2 \mathrm{BH} 2$ domain for the interaction of INrf2 with $\mathrm{Bcl}-2$ is 
a

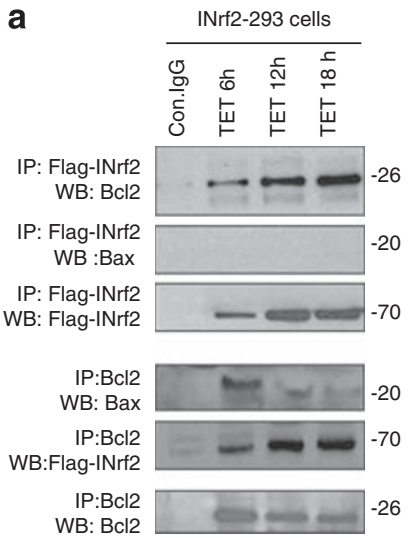

d

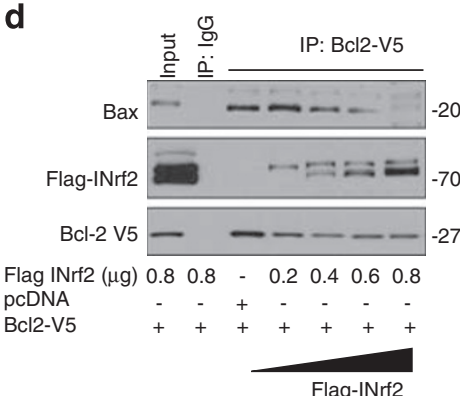

b

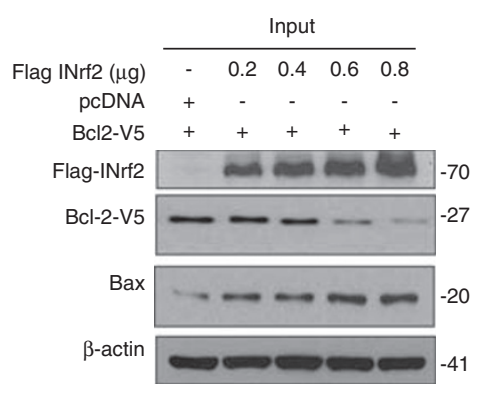

e

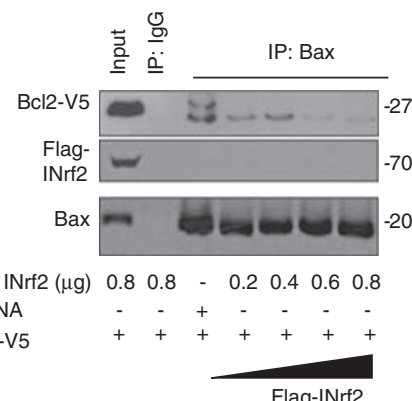

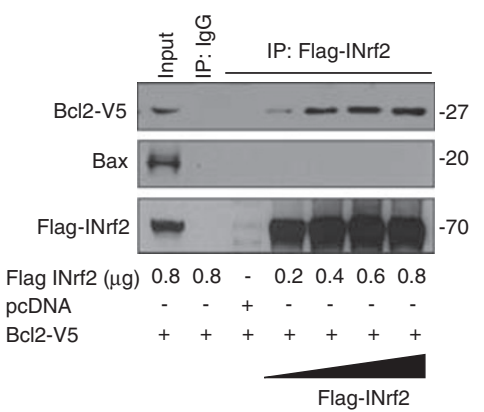

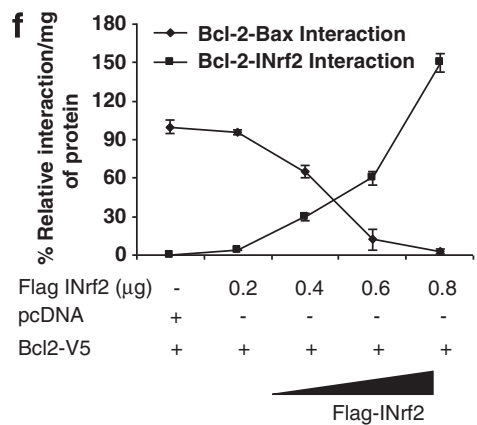

Figure 4 INrf2 decreases Bcl-2:Bax interaction. (a) IP/immunoblot analysis of Bcl-2 interaction with INrf2 and Bax. INrf2-293 cells were treated with tetracycline $(0.5 \mu \mathrm{g} / \mathrm{ml})$ for different time periods to express Flag-INrf2. The cells were harvested and $1 \mathrm{mg}$ of cell lysates was immunoprecipitated with anti-Flag or anti-Bcl-2 antibody and immunoblotted with anti-Bax, anti-Bcl2, and anti-Flag antibody. (b) Immunoblot analysis of the effect of overexpression of Flag-INrf2 on Bcl-2 and Bax. Hepa-1 cells were co-transfected with increasing concentrations of Flag-INrf2, along with the same concentration of Bcl-2-V5, and cell lysates were immunoblotted with indicated antibodies (input panel). (c-e) IP/immunoblot analysis of Bcl-2 interaction with INrf2 and Bax. A volume of $1 \mathrm{mg}$ of cell lysates from transfected Hepa-1 cells in 'b' was immunoprecipitated with either anti Flag-INrf2 (c) or anti-V5 (d) or anti-Bax (e) antibodies and immunoblotted with indicated antibodies. (f) The relative interaction of Bcl-2-V5:Bax and Bcl-2-V5:Flag-INrf2 was quantified from three experiments and plotted as mean \pm S.D.

also supported by immunofluorescence studies (Supplementary Figure S4a and b).

Overexpression of INrf2 decreases the Bcl-2-Bax complex. The $\mathrm{BH} 1$ and $\mathrm{BH} 2$ domains of $\mathrm{Bcl}-2$ heterodimerize with Bax to exert its antiapoptotic action. ${ }^{23} \mathrm{We}$ conducted experiments to investigate whether INrf2 disturbs the Bcl-2:Bax complex through degradation of Bcl-2. First, we analyzed the interaction of endogenous Bcl-2 and Bax after overexpression of Flag-INrf2 in INrf2-293 cells. Increasing Flag-INrf2 in INrf2-293 cells by tetracycline increased INrf2-Bcl-2 interaction. Interestingly, INrf2 failed to pull down endogenous Bax protein, indicating that INrf2 does not interact with Bax (Figure 4a, upper panels). In related experiments, the endogenous $\mathrm{Bcl}-2-\mathrm{Bax}$ interaction was significantly reduced when $\mathrm{Bcl}-2$ interaction with Flag-INrf2 was increased (Figure $4 \mathrm{a}$ lower panels). This was also supported by a dose-dependent overexpression of INrf2 in Hepa- 1 cells that degraded Bcl-2 and stabilized Bax protein as expected (Figure 4b). The immunoprecipitation of FlagINrf2 in the same experiment showed increased interaction of INrf2 with Bcl-2, but not with Bax (Figure 4c). Immunoprecipitation of Bcl-2-V5 with anti-V5 antibody demonstrated interaction of $\mathrm{Bcl}-2$ with both INrf2 and Bax (Figure 4d). Interestingly, increase in INrf2 interaction with $\mathrm{Bcl}-2$ led to a decrease in interaction between Bcl-2 and Bax (Figure 4d). This suggested that INrf2 decreases Bcl-2:Bax heterodimerization by the degradation of $\mathrm{Bcl}-2$. In the same experiment, immunoprecipitation of Bax showed interaction of Bax with Bcl-2 but not with INrf2 (Figure 4e), further supporting the above conclusions. Figure $4 \mathrm{f}$ shows that an increase in INrf2:Bcl-2 interaction leads to the decrease in $\mathrm{Bcl}-2$ :Bax interaction.

Antioxidant tert-butylhydroquinone (t-BHQ) treatment destabilized the Bcl-2:INrf2:Cul3-Rbx1 complex and increased $\mathrm{Bcl}-2 \mathrm{S70}$ phosphorylation and stabilization of Bcl-2. We hypothesized that antioxidants that antagonize INrf2:Nrf2 interaction would also antagonize INrf2:Bcl-2 interaction. Indeed, antioxidant $\mathrm{tBHQ}$ destabilized/degraded endogenous INrf2, which resulted in stabilized $\mathrm{Bcl}-2$ protein (Figure 5a, left panel). Immunoprecipitation of INrf2 from Hepa-1 cells treated with DMSO and t-BHQ (2h) showed decreased interaction of INrf2 with Cul3-Rbx1 and Bcl-2 in cells treated with $\mathrm{t}-\mathrm{BHQ}$, as compared with DMSO-treated control cells (Figure 5a, second left panel). Immunoprecipitation of $\mathrm{Cul} 3$ or $\mathrm{Rbx} 1$ showed a more or less similar interaction of Cul3 with Rbx1 between t-BHQ- and DMSOtreated cells. However, interaction with INrf2 and INrf2 binding $\mathrm{Bcl}-2$ proteins significantly decreased in cells 

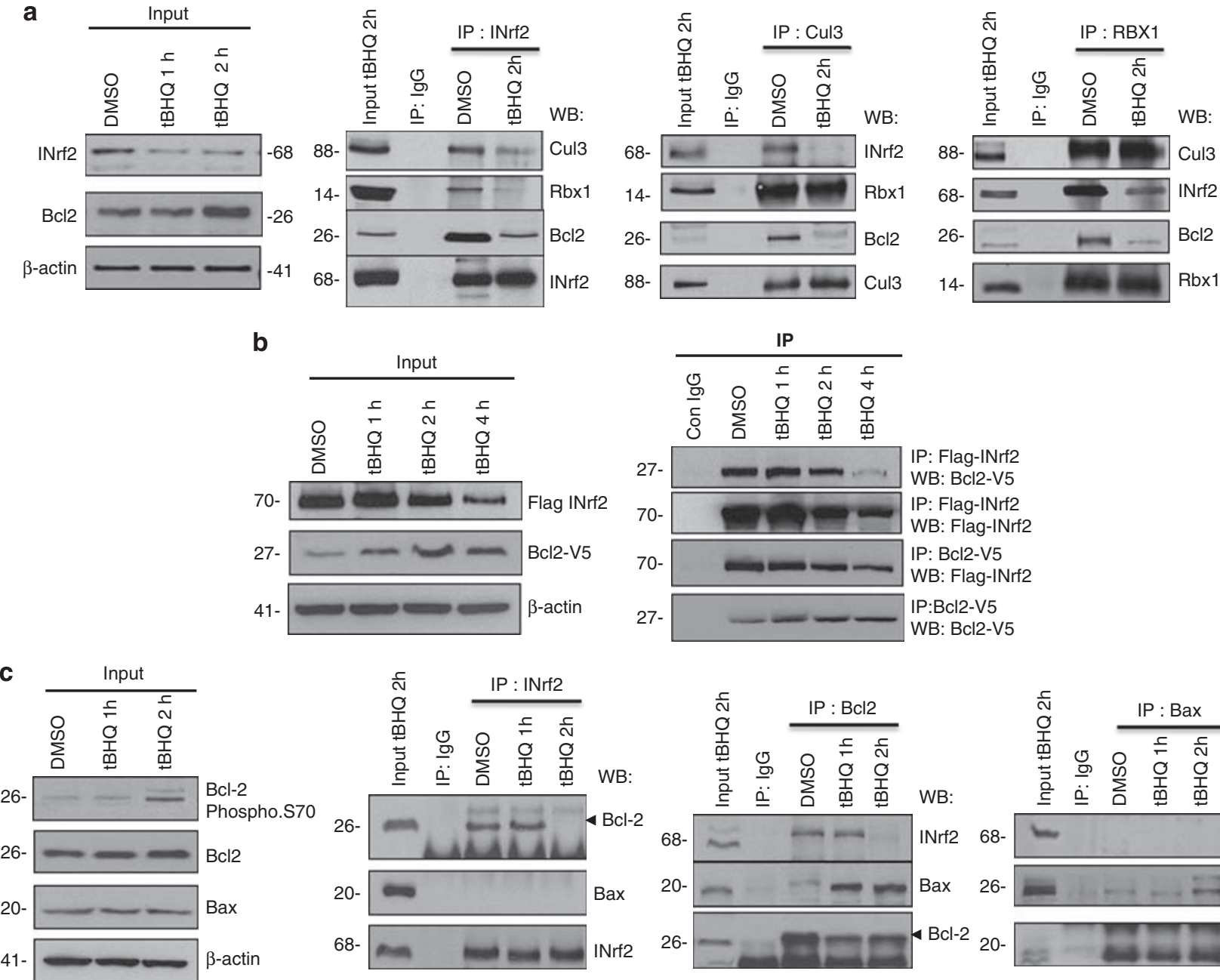

Figure $5 \mathrm{tBHQ}$ treatment destabilizes the Bcl-2:INrf2:Cul3-Rbx1 complex, stabilizes Bcl-2 and increases Bcl-2S70 phosphorylation and interaction with Bax. (a) IP/Immunoblot analysis. Hepa-1 cells were treated with DMSO or $\mathrm{tBHQ}(50 \mu \mathrm{M})$ for 1 or $2 \mathrm{~h}$ and $60 \mu \mathrm{g}$ of cell lysates was immunoblotted with antibodies as shown under input in the left panel. A volume of $1 \mathrm{mg}$ of Hepa-1 cell extracts treated with DMSO or t-BHQ(2 $\mathrm{h})$ from the input panel was immunoprecipited with anti-INrf2, anti-Cul3, and antiRbx1 antibodies and immunoblotted with antibodies as shown. (b). IP/Immunoblot analysis. Hepa-1 cells were co-transfected with Bcl-2-V5 and Flag-INrf2, treated with DMSO or tBHQ $(50 \mu \mathrm{M})$ for $1-4 \mathrm{~h}$, and $60 \mu \mathrm{g}$ of cell lysates was immunoblotted with indicated antibodies (input left panel). The same cell lysates (1 mg) were immunoprecipitated with anti-Flag or anti-V5 antibodies and immunoblotted with indicated antibodies (right panel). (c) IP/immunoblot analysis. Hepa-1 cells were treated with DMSO or tBHQ ( $50 \mu \mathrm{M})$ for 1 or $2 \mathrm{~h}$ and cell lysates were immunoblotted with antibodies as shown in the input left panel. A volume of $1 \mathrm{mg}$ of the same cell lysates from DMSO- and tBHQ-treated cells was immunoprecipited with either anti-INrf2 or anti-Bcl-2 or anti-Bax antibodies and immunoblotted with indicated antibodies as shown in the three panels on the right. We used $1 \mu \mathrm{g}$ and $2 \mu \mathrm{g}$ of INrf2 antibody for DMSO- and t-BHQ-treated samples, respectively. This was to keep INrf2 close to equal in immunoprecipitated samples for comparison of Bcl-2 interaction with INrf2. For Bcl-2 and Bax immunoprecipition, $1 \mathrm{mg}$ of cell lysates and $1 \mu \mathrm{g}$ of antibody were used. All experiments were conducted three times

treated with $\mathrm{t}-\mathrm{BHQ}$, as compared with DMSO-treated cells (Figure 5a, right two panels). In other words, treatment with $\mathrm{t}-\mathrm{BHQ}$ led to dissociation of the Cul3-Rbx1:INrf2:Bcl-2 complex, leading to degradation of INrf2 and stabilization of $\mathrm{Bcl}-2$. Similar results were also observed with Hepa-1 cells overexpressing Flag-INrf2 and Bcl-2-V5 (Figure 5b). t-BHQ treatment showed a time-dependent decrease in INrf2 and an increase in Bcl-2 (Figure 5b, left panel). Forward and reverse immunoprecipitation and immunoblot analysis revealed t-BHQ treatment time-dependent decreases in Flag-INrf2 and Bcl-2V5 interaction, degradation of Flag-INrf2, and stabilization of $\mathrm{Bcl}-2-\mathrm{V} 5$ (Figure $5 \mathrm{~b}$, left and right panels). Interestingly, t-BHQ treatment of Hepa-1 cells also increased endogenous Bcl-2-S70 phosphorylation (Figure 5c, left panel). Immunoprecipitation of
INrf2 from DMSO- and t-BHQ-treated cells showed t-BHQdependent release of Bcl-2 from INrf2, no interaction of Bax with INrf2, and increased interaction of Bcl-2 and Bax (Figure 5c, right panels). These data suggested that $\mathrm{tBHQ}$ destabilized the Cul3-Rbx1:INrf2:Bcl-2 complex, increased phosphorylation of Bcl-2S70, leading to dissociation of Bcl-2 from INrf2 and increased Bcl-2:Bax interaction.

INrf2 overexpression and etoposide treatment upregulate proapoptotic marker proteins and promote apoptosis. Hepa-1 cells were transfected and INrf2-293 cells treated with tetracycline to overexpress INrf2. This was followed by treatment with etoposide. INrf2 overexpression and etoposide treatments increased Bax protein by two- to 
a

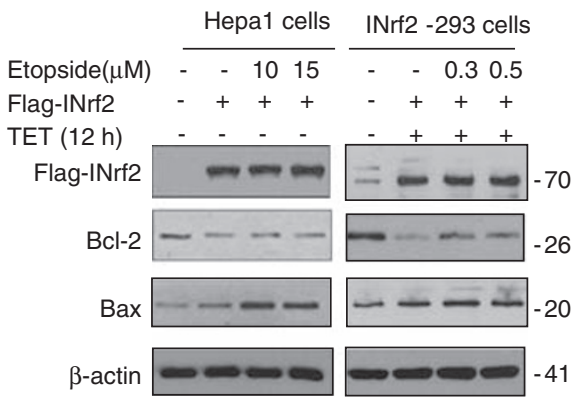

d

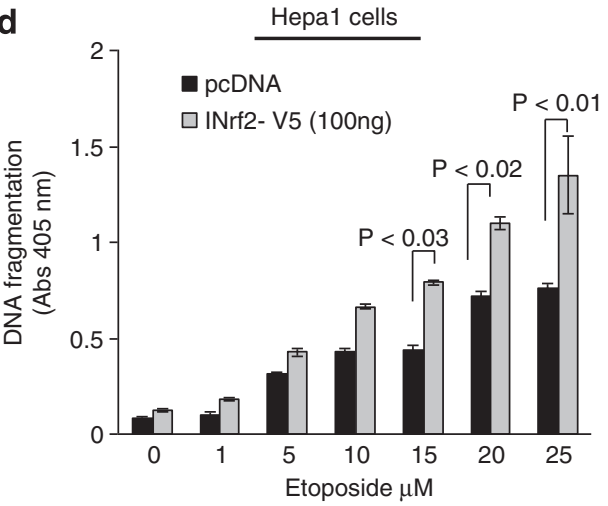

b

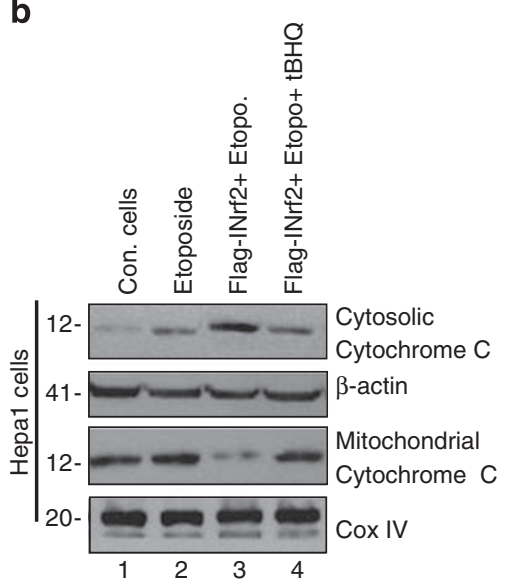

e

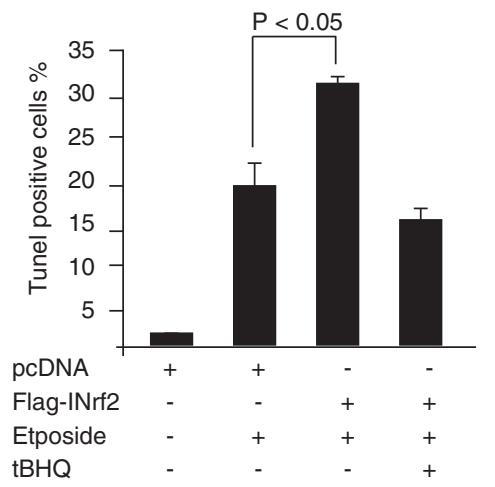

c

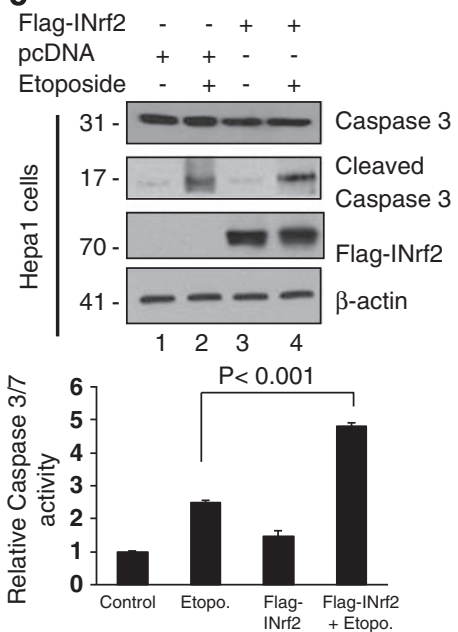

f

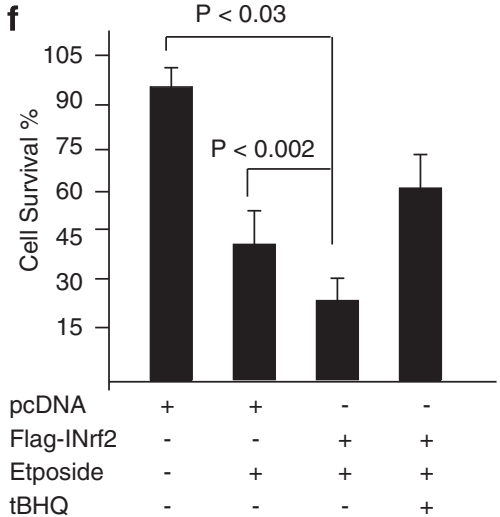

Figure 6 INrf2 overexpression and etoposide treatments upregulate proapoptotic marker proteins and induce cellular apoptosis. (a) Immunoblot analysis. Hepa-1 cells transfected with Flag-INrf2, or INrf2-293 cells treated with tetracycline were treated with etoposide as indicated and lysates were immunoblotted with antibodies as shown. (b) Immunoblot analysis. Hepa-1 cells were transfected with Flag-INrf2 and treated with etoposide $(30 \mu \mathrm{M})$ for $48 \mathrm{~h}$ or with etoposide plus tBHQ for $48 \mathrm{~h}$. Cells were harvested and mitochondria were isolated using a mitochondria isolation kit (Thermo Scientific, Rockford, IL USA) and $30 \mu \mathrm{g}$ of mitochondrial and cytosolic lysates was immunoblotted. (c) Immunoblot and activity analysis. Hepa-1 cells were transfected with pcDNA or Flag-INrf2 for $24 \mathrm{~h}$ and treated with and without $30 \mu \mathrm{M}$ etoposide for an additional $48 \mathrm{~h}$. A volume of $60 \mu \mathrm{g}$ of lysates was immunoblotted with caspase-3 and anti-Flag antibodies. Caspase-3/7 activity was measured from the same lysates using a caspase-3/7 Luminescent Assay Kit, Caspase-Glo(Promega), and plotted (lower panel). (d) DNA fragmentation assay. Hepa-1 cells transfected with pcDNA or INrf2-V5, treated with indicated concentrations of etoposide for $72 \mathrm{~h}$, and cytosolic DNA fragmentations were quantified using a Cell Death Detection ELISA kit (Roche) and plotted. (e) TUNNEL assay. Hepa-1 cells grown on coverslips were transfected with Flag-INrf2, and treated with etoposide $(30 \mu \mathrm{M} ; 30 \mathrm{~h})$ or etoposide $+\mathrm{tBHQ}(50 \mu \mathrm{M})$ for $24 \mathrm{~h}$. Cells were fixed, permeabilized, and TUNNEL assay was performed. TUNNEL-positive cells were quantified and plotted. (f) Cell survival assay. Hepa-1 cells transfected with Flag-INrf2 were treated with etoposide or etoposide and tBHQ for $72 \mathrm{~h}$, incubated with fresh MTT solution for $2 \mathrm{~h}$ and absorbance at $490 \mathrm{~nm}$ was measured. All experiments were repeated three times. Data were shown as the mean value \pm S.D. from three independent experiments

threefold and decreased Bcl-2 in both cell lines (Figure 6a). INrf2 overexpression, followed by etoposide treatment, induced cytochrome $c$ release from mitochondria to cytosol by 2.1-fold compared with etoposide treatment alone (Figure 6b, compare lanes 1/2/3). INrf2:etoposide-mediated induction of cytochrome $c$ in the cytosol was reversed by the posttreatment of cells with $\mathrm{tBHQ}$ (Figure 6b, compare lanes $3 / 4)$. The endogenous caspases- $3 / 7$ activity was stimulated by 1.45 -fold in Hepa-1 cells transfected with Flag-INrf2, compared with that in pcDNA-transfected control cells. Etoposide treatment itself increased caspase-3/7 activity by 2.8-fold (Figure 6c, lower panel). Interestingly, overexpression of Flag-INrf2 and etoposide treatment increases caspase- $3 / 7$ activity by 4.6 -fold, compared with control cells (Figure 6c, lower panel). Further, immunoblotting experiments confirmed that INrf2 activates caspase-3 by the cleavage of native caspase-3 protease. Significant amounts of cleaved caspase-3 peptide fragments were detected by immunoblotting after Hepa- 1 cells were transfected with Flag-INrf2 and treated with etoposide, compared with control cells (Figure 6c upper panel; compare lane 2 and 4). These results demonstrated that overexpression of INrf2 and etoposide treatments upregulate Bax protein, induce cytochrome $c$ release from mitochondria, and activate caspases $3 / 7$.

The biochemical significance of upregulation of proapoptotic marker proteins by INrf2 raised an interesting question of whether INrf2 modulated cellular apoptosis. Three assays were used to determine this. Hepa-1 cells transfected with pcDNA (control) or INrf2-V5 were treated with various 

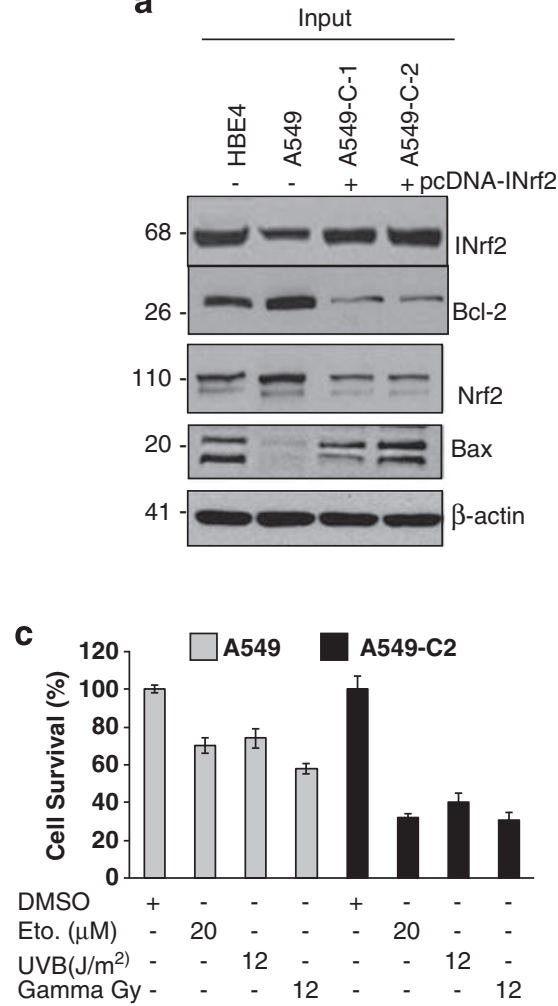
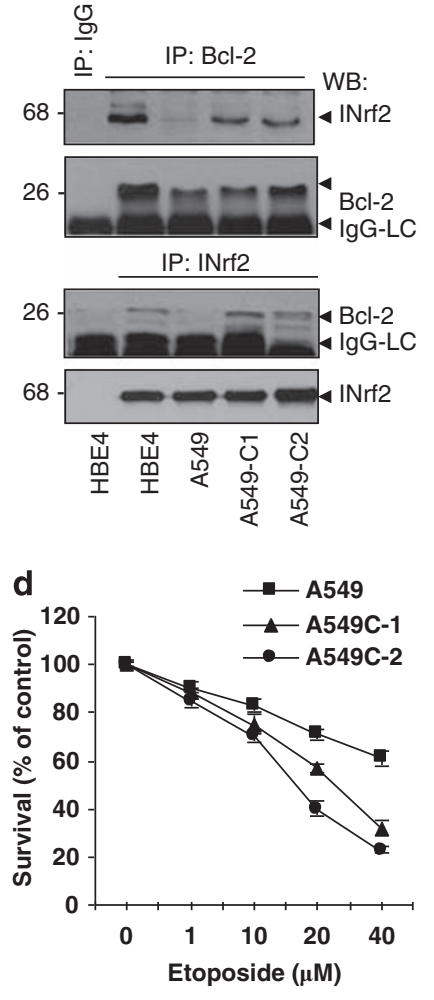

b

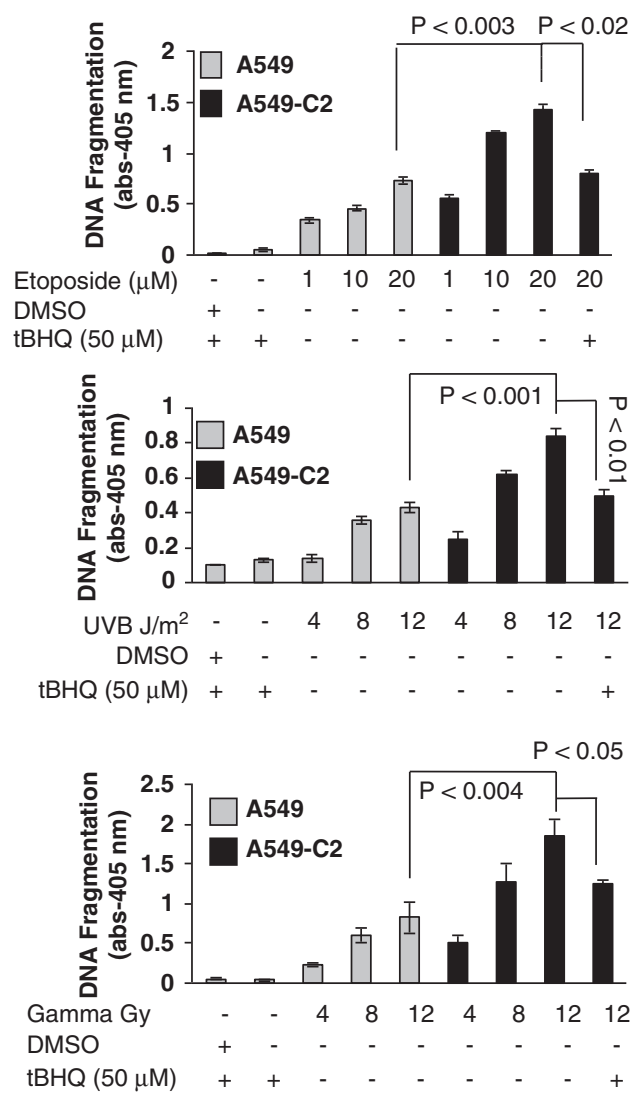

Figure 7 Dysfunctional INrf2 stabilized Bcl-2 and reduced etoposide and UV/ $\gamma$ radiation-mediated DNA fragmentation in non-small-cell lung cancer A549 cells. (a) Left panel: Immunoblot analysis of lung cancer HBE4 cells expressing normal INrf2, A549 expressing mutant INrf2 and A549-C1/C2 expressing cDNA-derived wild-type INrf2. A volume of $60 \mu \mathrm{g}$ of cell lysates was immunoblotted with anti-INrf2, anti- Bcl-2, anti Nrf2, and anti-Bax antibodies. Right panels: IP/immunoblot analysis demonstrating INrf2 and $\mathrm{Bcl}-2$ interaction in normal HEB4 and lung cancer cells A549, A549-C1, and A549-C2. A volume of $1 \mathrm{mg}$ of lysates from the input left panel was immunoprecipitated with control IgG or anti-Bcl-2 or anti-INrf2 antibody and immunoblotted with indicated antibodies. (b) Apoptosis assay. A549 and A549-INrf2-C2 cells were treated with etoposide for $48 \mathrm{~h}$ or exposed to UVB or $\gamma$ radiation as indicated in figures. One set of cells was further treated with $\mathrm{HBHQ}(50 \mu \mathrm{M})$ for an additional $24 \mathrm{~h}$. The cytoplasmic histone-associated DNA fragments (mono and oligonucleosomes) were quantified using a Cell Death Detection ELISA kit (Roche) and plotted. (c) MTT Cell survival assay. A549 or A549-INrf2-C2 cells were treated with etoposide or exposed to UV or $\gamma$ radiation and cell survival was measured by MTT assay as described in Materials and methods. (d) Clonogenic cell survival assay. A549, A549-C1, A549-C2 cells were grown to 70\% confluence and exposed to DMSO or different concentrations of etoposide (1-40 $\mu \mathrm{M})$ for $48 \mathrm{~h}$. Cells were treated with trypsin and 1000 cells were re-seeded in $100 \mathrm{~mm}$ tissue culture dishes (in triplicate) and incubated for 9 days. A freshly prepared clonogenic reagent was added to the plates and blue colonies were counted. The experiment was repeated twice. Each data point represents a mean \pm S.D. and was normalized to the value of the corresponding control cells

concentrations of etoposide and analyzed for histoneassociated DNA fragmentation (Figure $6 \mathrm{~d}$ ). The results demonstrated that overexpression of INrf2, followed by etoposide treatments, enhanced DNA fragmentation at least 1.4- to 2-fold, compared with control cells. The TUNNEL assay supported the results from the DNA fragmentation assay. TUNNEL-positive cells were photographed (Supplementary Figure S5) and counted/plotted (Figure 6e). Hepa-1 cells overexpressing INrf2 on treatment with etoposide showed $15 \%$ more TUNNEL-positive cell population, as compared with etoposide-treated control cells (Figure 6e and Supplementary Figure S5). $\mathrm{BBHQ}$ treatment protected the cells from etoposide-mediated DNA damage as the TUNNEL positive cells declined (Figure $6 e$ and Supplementary Figure S5). DNA fragmentation/TUNNEL data were also supported by cell survival assay (Figure 6f). Overexpression of Flag-INrf2 in Hepa-1 cells significantly reduced cell survival on treatment with etoposide, as compared with etoposidetreated control cells expressing endogenous levels of INrf2 (Figure 6f). t-BHQ treatment, as in the case of TUNNEL assay, increased survival of cells in the presence of etoposide (Figure $6 e$ and $f$ and Supplementary Figure S5). Flow cytometry (FACS) analysis also suggested that INrf2 overexpression alone in INrf2-293 cells significantly increased $(\sim 10 \%)$ apoptotic cell population, as well as etoposidemediated DNA fragmentation (Supplement Figure S6a-c).

Dysfunctional INrf2 in lung cancer cells stabilized Bcl-2, and decreased etoposide or $U \mathrm{~V} / \gamma$ radiation-mediated DNA fragmentation, and increased cell survival. HBE4 (human lung) and A549 (human non-small-cell lung cancer) cells that express wild-type INrf2 and mutant INrf2G333C, respectively, were used. ${ }^{13}$ Mutant INrf2G333C lacks the capacity to degrade/repress Nrf2. Therefore, A549 cells 
carrying mutant INrf2G333C showed accumulation of Nrf2 and are resistant to drugs. ${ }^{13}$ Glycine 333 residue is present in the first Kelch (DGR) domain of INrf2 to which Bcl-2 binds as shown earlier in this study. This raised interesting questions regarding the capacity of mutant INrf2G333C to bind and degrade Bcl-2. Two clones (A549-C-1 and A549-C-2) expressing wild-type INrf2 were generated. Immunoblot analysis of HBE4, A549, A549-C-1, and A549-C-2 cells is shown in Figure 7a (left panel). A549 cells expressing mutant INrf2G333C protein showed accumulation of $\mathrm{Bcl}-2$ and Nrf2, and almost no Bax, as compared with HBE4 cells. Interestingly, A549-C-1 and C-2 clones expressing mutant and wild-type INrf2 protein demonstrated a decrease in Bcl-2 and Nrf2, and an increase in Bax, as compared with A549 cells expressing mutant alone. Immunoprecipitation of $\mathrm{Bcl}-2$ (Figure 7a, right upper two panels) or INrf2 (Figure 7a, right lower two panels) demonstrated more or less an absence of interaction between mutant INrf2G333C and Bcl-2. Both A549-stabilized clones expressing wild-type INrf2 showed interaction between INrf2 and Bcl-2 proteins (Figure 7a, right panels). Consistent with INrf2-Bcl-2 interaction, etoposidemediated DNA fragmentation in A549 cells was twofold lower compared with that in the A549-C-2 clone (Figure 7b, upper panel). $t-B H Q$ treatment, as expected, reduced DNA fragmentation in A549-C-2 cells (Figure 7b). In addition, A549 cells expressing endogenous mutant INrf2 exposed to UVB and $\gamma$ radiation showed significantly ( $\sim 2.2$-fold) lower DNA fragmentation compared with A549-C-2 cells expressing endogenous mutant and cDNA-derived wildtype INrf2 (Figure 7b, middle and lower panels). Further, A549 cells showed increased $(\sim 20-30 \%)$ cell survival compared with the A549-C2 clone when exposed to drug or radiation (Figure $7 \mathrm{c}$ ). In addition, we compared the relative cell survival in A549, A549-C-1, and A459-C-2 cells by clonogenic cell survival assay after treatment of cells with different concentrations of etoposide. The data demonstrate that A549-C-1 and A549-C-2 cells show 35 and $40 \%$ reduction in cell survival, respectively, compared with $A 549$ cells (Figure 7d). These data together suggest that point mutation in the DGR region of INrf2 reduced INrf2 interaction with $\mathrm{Bcl}-2$, resulted in stabilization of $\mathrm{Bcl}-2$, decreased drug or $\mathrm{UV} / \gamma$ radiation-induced DNA fragmentation, and promoted cell survival.

\section{Discussion}

We demonstrated that INrf2 directly interacts with Bcl-2 and functions as an adaptor protein for Cul3-Rbx1-mediated ubiquitination of $\mathrm{Bcl}-2$-lysine 17 residue and degradation of $\mathrm{Bcl}-2$. Domain-mapping studies demonstrated that the DGR domain of INrf2 and the $\mathrm{BH} 2$ domain of $\mathrm{Bcl}-2$ were required for their interaction. Overexpression of INrf2 led to degradation of $\mathrm{Bcl}-2$ and decreased $\mathrm{Bcl}-2: \mathrm{Bax}$ heterodimers. The treatment of INrf2-overexpressing cells with etoposide upregulated Bax protein, induced cytochrome $c$ release from mitochondria, activated caspase-3/7, and led to increased apoptotic cell death. In other words, increase in INrf2 promoted apoptosis by degrading $\mathrm{Bcl}-2$. On the other hand, antioxidant treatment antagonized INrf2:Bcl-2 interaction, led to stabilization of $\mathrm{Bcl}-2$, increased interaction of $\mathrm{Bcl}-2$ with $\mathrm{Bax}$, and decreased etoposide-mediated cellular apoptosis. Together, these results concluded that INrf2 regulates apoptotic cell death by controlling antiapoptotic protein Bcl-2. It is noteworthy that INrf2 is known to bind to PGAM5, which binds to $\mathrm{Bcl}-\mathrm{xL} .{ }^{24}$ Therefore, the role of INrf2 in regulation of $\mathrm{Bcl}-\mathrm{xL}$ through PGAM5 in apoptosis is also expected but remains unknown.

The mechanism of dissociation of Bcl-2 from INrf2 remains unknown. However, it is possible that oxidative/electrophilic modification of INrf2C151 and phosphorylation of Bcl-2 are required for the dissociation of $\mathrm{Bcl}-2$ from INrf2. This assumption is based on INrf2:Nrf2 studies. ${ }^{7,9}$ Our data showed that antioxidants increased Bcl-2S70 phosphorylation. The role of this phosphorylation in the release of $\mathrm{Bcl}-2$ from INrf2 is possible but remains unknown. It is noteworthy that Bcl2S70 phosphorylation is required for heterodimerization with Bax and cell survival. ${ }^{25-27}$ The release of $\mathrm{Bcl}-2$ from INrf2 and prevention of apoptosis are presumably important mechanisms that protect cells from dying in acute stress. Once the exposure effect subsides, the level of $\mathrm{Bcl}-2$ protein is brought back to normal.

Recent studies have reported increased stabilization/ accumulation of Nrf2 because of mutations in INrf2, resulting in loss of function in lung and breast tumors. ${ }^{12,13,28,18}$ The lung cancer cell line A549 contains the INrf2G333C mutant protein that has lost its capacity to bind/degrade Nrf2, leading to accumulation of Nrf2. ${ }^{13}$ It has been suggested that higher levels of Nrf2 in A549 cells might have contributed to the survival of these cells in lung cancer. Our studies demonstrate that INrf2G333C mutant is also incapable of binding/degrading Bcl-2 and this must also have contributed to the survival of A549 cells. This is also supported by observations from this study that expression of wild-type INrf2 in A549 cells reduced $\mathrm{Bcl}-2$ and increased etoposide-mediated apoptosis. The present studies also demonstrated that Bcl-2 accumulation and reduced apoptosis in lung cancer A549 cells containing mutant INrf2 protein contributed to drug (etoposide) or radiation resistance.

In conclusion, INrf2 functions as an adaptor for the Cul3Rbx1-mediated ubiquitination and degradation of $\mathrm{Bcl}-2$ as depicted in a model (Supplementary Figure S7). INrf2 under normal conditions facilitates Cul3-Rbx1-mediated ubiquitination and degradation of $\mathrm{Bcl}-2$. This contributes to the maintenance of normal level of Bcl-2:Bax heterodimers and apoptosis. Oxidative/electrophilic stresses lead to dissociation of $\mathrm{Bcl}-2$ from INrf2, causing cellular levels of $\mathrm{Bcl}-2$ to increase. This leads to an increase in Bcl-2:Bax heterodimers, a decrease in Bax, reduced apoptosis, enhanced cell survival, and drug resistance.

\section{Materials and methods}

Plasmid constructs. A full-length mouse INrf2 coding sequence (1872 bp) and various deletion domains of INrf2 were cloned into GFP vector as described earlier. ${ }^{1}$ We also amplified various deletions of the DGR region of INrf2 by PCR and cloned into $\triangle$ DGR-INrf2-GFP constructs. Mouse Bcl-2 cDNA purchased from Addgene (Cambridge, MA, USA) was subcloned into pcDNA3.1/V5-His/Topo vector and also into pcmx-Flag vector. We constructed Flag- $\Delta \mathrm{BH} 4-\mathrm{Bcl}-2$, Flag- $\Delta \mathrm{BH} 3-\mathrm{Bcl}-$ 2, Flag- $\triangle \mathrm{BH} 2-\mathrm{Bcl}-2$, and Flag- $\Delta \mathrm{BH} 1-\mathrm{Bcl}-2$ deletion domains of $\mathrm{BCl}-2$ using two steps, PCR and ligation. V5-tagged Bcl2K17A, Bcl-2K22A, Bcl-2K214A, Bcl-2K236A, Bcl-2W185A, and Bcl-2G190AG191A mutants were generated by using a Gene Tailor site-directed mutagenesis Kit (Invitrogen, Carlsbad, CA, USA). 
The primer sequences used for construction of various INrf2 and Bcl-2 constructs are shown in Supplementary Table 1. The construction of pcmx-Flag-INrf2, pcDNAINrf2-V5, and HA-UB has been described previously. ${ }^{1}$ All plasmids were confirmed by DNA sequencing.

Cell culture. Hepa-1 cells and HEK293 cells were grown in DMEM medium supplemented with $10 \%$ FBS and antibiotics. Generation of stable Flp-In T-REx HEK293 cells expressing tetracycline-inducible Flag-INrf2 was described previously. ${ }^{1}$ Lung cancer cell A549 (non-small-cell lung cancer) was grown in F12/DMEM medium. We also generated wild-type INrf2 expressing stable A549 cells by transfection of pcDNA-INrf2, followed by selection of clones with neomycin (G148). The cells were grown in monolayer in an incubator at $37^{\circ} \mathrm{C}$ in $95 \%$ air and $5 \% \mathrm{CO}_{2}$.

Co-immunoprecipitation and western blotting. Preparation of cell extracts, immunoblotting and co-immunoprecipitation methods have been described previously. ${ }^{7}$ The antibodies used and its dilution were anti-INrf2 (E-20) $(1: 1000)$, anti-Bcl-2 (N-19) $(1: 1000)$, anti-Bax (P-19) $(1: 1000)$, and anticytochrome $c(1: 1000)$. These antibodies were purchased from Santa Cruz Biotechnology (Santa Cruz, CA, USA). Other antibodies used were: antiphospho-Bcl-2S70, anti-Cul3, and anti-caspase 3 antibody from Cell Signaling (Danvers, MA, USA); anti-Cox IV from Abcam (Cambridge, MA, USA); anti-FlagHRP, anti-HA-HRP, and anti-actin from Sigma-Aldrich (St. Louis, MO, USA); antiGFP, anti-V5 and anti-V5-HRP antibodies from Invitrogen; and anti-Rbx1 antibody from Bio-Source (Camarillo, CA, USA). The intensity of the protein bands after western blotting was quantitated by using QuantityOne 4.6.3 Image software (ChemiDoc XRS, Bio-Rad).

Ubiquitination assay. Hepa-1 cells were co-transfected with $0.5 \mu \mathrm{g} \mathrm{HA}-\mathrm{Ub}$ and wild-type Bcl2-V5 construct or with various mutant constructs for $30 \mathrm{~h}$. Cell extracts $(1 \mathrm{mg})$ were immunoprecipitated with anti-Bcl-2 antibody or anti-V5 antibody. In some experiments, Bcl2-His-tagged protein was also pulled down by Ni-NTA resin and western blotted with anti HA-tagged or antiubiquitin antibodies as shown in figures. In in vitro ubiquitination assay, the in vitro-translated Bcl-2-V5 and lysine mutants or bacterially purified Bcl-2 proteins were incubated with $2 \mu \mathrm{g}$ of ubiquitin, $5 \mathrm{mM} \mathrm{MgCl}$, $2 \mathrm{mM}$ ATP in Tris/HCl buffer $\mathrm{pH}$ 8, along with overexpressed INrf2, Cul3, Rbx1 Hepa1 cell lysates $(20 \mu \mathrm{g})$ for $60 \mathrm{~min}$ at $37^{\circ} \mathrm{C}$. The ubiquitination reaction mixtures were equilibrated with RIPA buffer $(0.5 \mathrm{ml})$ and wild type and mutant $\mathrm{Bcl}-2$ proteins were immunoprecipitated with anti-V 5 antibody and western blotted with antiubiquitin antibody.

Plasmid DNA and siRNA transfection. Hepa-1 cells or HEK-293 cells were plated in $100 \mathrm{~mm}$ plates and transfected with $1 \mu \mathrm{g}$ of the indicated plasmids using Effectene transfection reagent (Qiagen, Valencia, CA, USA) according to the manufacturer's instructions. For siRNA transfection, Hepa-1 cells were transfected with control siRNA (GAPDH) or INrf2 siRNA or Cul3 siRNA using Lipofectamine RNAiMAX reagent (Invitrogen) according to the manufacturer's instructions. At $32 \mathrm{~h}$ after transfection, cells were harvested and endogenous proteins were analyzed by immunoblotting.

Real-time PCR. Hepa-1 cells were seeded in 100-mm plates. After $24 \mathrm{~h}$, cells were transfected with increasing concentrations of INrf2-V5 construct or endogenous INrf2 knockdown by siRNA $(25-50 \mathrm{nM})$ for $30 \mathrm{~h}$ and harvested. RNA was extracted using an RNeasy mini kit (Qiagen). RNA was converted into cDNA using a High Capacity cDNA Reverse Transcription kit (Applied Biosystems, Foster City, CA, USA) according to the manufacturer's instructions. cDNA was used with Taqman Master Mix (Applied Biosystems) and Bcl2 Primer and Probe amplicon (ID: Mm00477531_m1) or GusB amplicon Mm00446953_m1 as a control (Applied Biosystems). The total mix was run on a 7500 Real-Time System (Applied Biosystems) using relative quantitation according to the manufacturer's instructions.

DNA fragmentation and TUNNEL assay. Hepa-1 cells were plated at a density of 2000 cells per well in 96 -well plates. After $20 \mathrm{~h}$, cells were transfected with pcDNA or INrf2-V5 construct for $12 \mathrm{~h}$; thereafter, cells were exposed to various concentrations of etoposide for $72 \mathrm{~h}$. A549 cells or A549-C2 cells were also treated with etoposide or exposed to UVB or $\gamma$ radiation as indicated by different energies. Cells were further incubated for $30 \mathrm{~h}$ and harvested. A photometric enzyme immunoassay was performed for the quantitative in vitro determination of cytoplasmic histone-associated DNA fragments (mono and oligonucleosomes) after etoposide exposure to cells using a Cell Death Detection ELISA kit (Roche Dignostics, Indianpolis, IN, USA) and as per the manufacturer's instructions.
For TUNNEL assay, a Dead End Fluorometric TUNNEL assay kit (Promega, Madison, WI, USA) was used as per the manufacturer's protocol.

MTT cell survival assay. Hepa- 1 cells were plated at a density of 5000 cells per well in 96-well plates, transfected with Flag-INrf2 and exposed to etoposide $(10 \mu \mathrm{M})$ for $30 \mathrm{~h}$ and further treated with $\mathrm{DMSO}$ or $\mathrm{BHH}$ for an additional $24 \mathrm{~h}$. A549 or A549-C2 cells were exposed to etoposide or UVB or $\gamma$ radiation as indicated. Cells were incubated with fresh MTT solution $(200 \mu \mathrm{l} / \mathrm{well}$; stock $5 \mathrm{mg} / \mathrm{ml}$ in phosphate-buffered saline (PBS)) for $2 \mathrm{~h}$ and absorbance at $490 \mathrm{~nm}$ was measured. Each experiment was repeated three times. Each data point represents a mean \pm S.D. and is normalized to the value of the corresponding control cells.

Clonogenic cell survival assay. A549, A549-C1, and A549-C2 cells were grown to $70 \%$ confluence and exposed to DMSO or different concentrations of etoposide $(1-40 \mu \mathrm{M})$ for $48 \mathrm{~h}$. Cells were trypsinized and 1000 cells were reseeded in $100 \mathrm{~mm}$ tissue culture dishes (in triplicate) and incubated for 9 days. Fresh medium was added on day 5 . After 10 days of incubation, a freshly prepared $2 \mathrm{ml}$ clonogenic reagent ( $0.25 \% 1,9$ dimethyl-methylene blue in $50 \%$ ethanol) was added to the plates and plates were kept at room temperature for $45 \mathrm{~min}$. Cells were washed with PBS twice and blue colonies were counted. The experiment was repeated three times. Each data point represents a mean \pm S.D. and is normalized to the value of the corresponding control cells.

Statistical analyses. Differences between groups were determined using the one-way ANOVA test and SPSS-16 Software. Error bars indicate standard error of the means (S.E.M.) of triplicate samples and comparisons were made using the two-tailed Student's $t$-test for repeated measures. Differences between means were accepted as statistically significant at the 95\% level $(P<0.04)$.

\section{Conflict of Interest}

The authors declare no conflict of interest.

Acknowledgements. This work was supported by NIH Grant R01 ES012265. We thank our colleagues for helpful discussions.

1. Niture SK, Jaiswal AK. Prothymosin-á mediates nuclear import of INrf2: Cul3.Rbx1 complex to degrade nuclear Nrf2. J Biol Chem 2009; 284: 13856-13868.

2. Cullinan SB, Gordan JD, Jin J, Harper JW, Diehl JA. The Keap1BTB protein is an adaptor that bridges Nrf2 to a Cul3-based E3 ligase: Oxidative stress sensing by a Cul3-Keap1 ligase. Mol Cell Biol 2004; 24: 8477-8486.

3. Kobayashi A, Kang MI, Okawa H, Ohtsuji M, Zenke Y, Chiba T et al. Oxidative stress sensor Keap1 functions as an adaptor for $\mathrm{Cul3}$ based E3 ligase to regulate proteasomal degradation of Nrf2. Mol Cell Biol 2004; 24: 7130-7139.

4. Zhang DD, Lo SC, Cross JV, Templeton DJ, Hannink M. Keap1 is a redox regulated substrate adaptor protein for a Cul3-dependent ubiquitin ligase complex. Mol Cell Biol 2004; 24: 10941-10953.

5. Wakabayashi N, Dinkova-Kostova AT, Holtzclaw WD, Kang MI, Kobayashi A, Yamamoto M et al. Protection against electrophile and oxidant stress by induction of the phase 2 response: fate of cysteines of the Keap1 sensor modified by inducers. Proc Natl Acad Sci USA 2004; 101: 2040-2045.

6. Eggler AL, Liu G, Pezzuto JM, van Breemen RB, Mesecar AD. Modifying specific cysteines of the electrophile-sensing human Keap1 protein is insufficient to disrupt binding to the Nrf2 domain Neh2. Proc Natl Acad Sci USA 2005; 102: 10070-10075.

7. Niture SK, Jain AK, Jaiswal AK. Antioxidant-induced modification of INrf2C151 and PKC delta-mediated phosphorylation of Nrf2S40 both required for stabilization and nuclear translocation of Nrf2 and increased drug resistance. J Cell Sci 2009; 122: 4452-4464.

8. Kwak M, Wakabayashi N, Itoh K, Motohashi H, Yamamoto M, Kensler TW. Modulation of gene expression by cancer chemopreventive dithiolethiones through the Keap1 Nrf2 pathway: identification of novel gene clusters for cell survival. J Biol Chem 2003; 278 : $8135-8145$.

9. Niture SK, Kaspar JW, Shen J, Jaiswal AK. Nrf2 signaling and cell survival. Toxicol Appl Pharmacol 2010; 244: 37-42.

10. Jain AK, Jaiswal AK. GSK-3beta acts upstream of Fyn kinase in regulation of nuclear export and degradation of NF-E2 related factor 2. J Biol Chem 2007; 282: 16502-16510.

11. Wakabayashi N, Itoh K, Wakabayashi J, Motohashi H, Noda S, Takahashi S et al. Keap1-null mutation leads to postnatal lethality due to constitutive Nrf2 activation. Nat Genet 2003; 35: 238-245. 
12. Padmanabhan B, Tong KI, Ohta T, Nakamura Y, Scharlock M, Ohtsuji M et al. Structural basis for defects of Keap1activity provided by its point mutations in lung cancer. $\mathrm{Mol} \mathrm{Cell}$ 2006; 21: 689-700.

13. Singh A, Misra V, Thimmulappa RK, Lee H, Ames S, Hoque MO et al. Dysfunctional Keap1 Nrf2 interaction in non-small-cell lung cancer. PLos Med 2006; 3: 1865-1876.

14. Lee O, Jain AK, Papusha V, Jaiswal AK. An auto-regulatory loop between stress sensors INrf2 and Nrf2 controls their cellular abundance. J Biol Chem 2007; 282: 36412-36420.

15. Wang W, Chan JY. Nrf1 is targeted to the endoplasmic reticulum membrane by an $\mathrm{N}$-terminal transmembrane domain. Inhibition of nuclear translocation and transacting function. J Biol Chem 2006; 281: 19676-19687.

16. Karapetian RN, Evstafieva AG, Abaeva IS, Chichkova NV, Filonov GS, Rubtsov YP et al. Nuclear oncoprotein prothymosin alpha is a partner of Keap1: implications for expression of oxidative stress-protecting genes. Mol Cell Biol 2005; 25: 1089-1099.

17. Strachan GD, Morgan KL, Otis LL, Caltagarone J, Gittis A, Bowser R et al. Fetal Alz-50 clone 1 interacts with the human orthologue of the Kelch-like Ech-associated protein. Biochemistry 2004; 43: 12113-12122.

18. Lee D, Kuo K, Liu M, Chou C, Xia W, Du Y et al. Keap1 E3 ligase-mediated downregulation of NF- $\kappa$ B signaling by targeting IKK $\beta$. Mol Cell 2009; 36: 131-140.

19. Cory S, Adams JM. The Bcl-2 family: regulators of the cellular life-or-death switch. Nat Rev Cancer 2002; 2: 647-656.
20. Danial NN, Korsmeyer SJ. Cell death: critical control points. Cell 2004; 116: 205-219.

21. Giacomello M, Drago I, Pizzo P, Pozzan T. Mitochondrial $\mathrm{Ca}^{2+}$ as a key regulator of cell life and death. Cell Death Differ 2007; 14: 1267-1274.

22. Rong $\mathrm{Y}$, Distelhorst $\mathrm{CW}$. Bcl-2 protein family members: versatile regulators of calcium signaling in cell survival and apoptosis. Annu Rev Physiol 2008; 70: 73-91.

23. Yin XM, Oltvai $\mathrm{ZN}$, Korsmeyer $\mathrm{SJ}$. $\mathrm{BH} 1$ and $\mathrm{BH} 2$ domains of $\mathrm{Bcl}-2$ are required for inhibition of apoptosis and heterodimerization with Bax. Nature 1994; 369: 321-323.

24. Lo SC, Hannink M. PGAM5, a Bcl-XL-interacting protein, is a novel substrate for the redox-regulated Keap1-dependent ubiquitin ligase complex. J Biol Chem 2006; 281 : 37893-37903.

25. Ito T, Deng X, Carr B, May WS. Bcl-2 phosphorylation required for anti-apoptosis function. J Biol Chem 1997; 272: 11671-11673.

26. Mai H, May WS, Gao F, Jin Z, Deng X. A functional role for nicotine in $\mathrm{Bcl} 2$ phosphorylation and suppression of apoptosis. J Biol Chem 2003; 278: 1886-1891.

27. Deng X, Gao F, Flagg T, Anderson J, May SW. Bcl2's Flexible Loop Domain Regulates p53 Binding and Survival. Mol Cell Biol 2006; 26: 4421-4434.

28. Kweon MH, Adhami VM, Lee JS, Mukhtar H. Constitutive overexpression of Nrf2dependent heme oxygenase- 1 in A549 cells contributes to resistance to apoptosis induced by epigallocatechin 3-gallate. J Biol Chem 2006; 281: 33761-33772.

Supplementary Information accompanies the paper on Cell Death and Differentiation website (http://www.nature.com/cdd) 


\title{
Retraction
}

\section{Retraction: INrf2 (Keap1) targets Bcl-2 degradation and controls cellular apoptosis}

\author{
SK Niture and AK Jaiswal
}

Cell Death and Differentiation advance online publication, 13 December 2013; doi:10.1038/cdd.2013.185

Retraction to: Cell Death and Differentiation (2011) 18, 439-451; doi:10.1038/cdd.2010.114

Although majority of the data are reproducible and conclusions do not change, as they are supported by several experiments, the corresponding author has decided to retract this article in its entirety due to reuse of Figure 5 a from other publication by the first author. 